\title{
On the large-scale structure and spectral dynamics of two-dimensional turbulence in a periodic channel
}

\author{
W. Kramer, H. J. H. Clercx, ${ }^{a)}$ and G. J. F. van Heijst \\ Fluid Dynamics Laboratory, ${ }^{b}$ Department of Physics, Eindhoven University of Technology, \\ P.O. Box 513, 5600 MB Eindhoven, The Netherlands
}

(Received 25 September 2007; accepted 3 April 2008; published online 20 May 2008)

\begin{abstract}
This paper reports on a numerical study of forced two-dimensional turbulence in a periodic channel with flat no-slip walls. Since corners or curved domain boundaries, which are met in the standard rectangular, square, or circular geometries, are absent in this geometry, the (statistical) analysis of the flow is substantially simplified. Moreover, the use of a standard Fourier-Chebyshev pseudospectral algorithm enables high integral-scale Reynolds number simulations. The paper focuses on (i) the influence of the aspect ratio of the channel and (ii) the integral-scale Reynolds number on the large-scale self-organization of the flow. It is shown that for small aspect ratios, a unidirectional flow spontaneously emerges, notably in the absence of a pressure gradient in the longitudinal direction. For larger aspect ratios, the flow tends to organize into an array of counter-rotating vortical structures. The computed energy and enstrophy spectra provide further evidence that the injection of small-scale vorticity at the no-slip walls modify the inertial-range scaling. Additionally, the quasistationary final state of decaying turbulence is interpreted in terms of the Stokes modes of a viscous channel flow. Finally, the transport of a passive tracer material is studied with emphasis on the role of the large-scale flow on the dispersion and the spectral properties of the tracer variance in the presence of no-slip boundaries. () 2008 American Institute of Physics. [DOI: 10.1063/1.2919132]
\end{abstract}

\section{INTRODUCTION}

Since the pioneering works on two-dimensional (2D) turbulence by Kraichnan, ${ }^{1}$ Batchelor, ${ }^{2}$ and Leith, ${ }^{3}$ many theoretical and numerical studies have been conducted to unravel the dynamics of $2 \mathrm{D}$ turbulence. The strong interest in understanding the dynamics of such 2D flows is due to the fact that many three-dimensional (3D) flows such as those encountered in the oceans and in the atmosphere, and also in many laboratory experiments, can under certain circumstances behave in a quasi-2D manner. There are several physical reasons why the large-scale flow dynamics in, for example, the atmosphere or oceans is behaving quasi-twodimensionally. Such geophysical flows have horizontal scales of hundreds of kilometers in the ocean and of an order of thousands of kilometers in the atmosphere, while their vertical extension measures a few kilometers only. One of the reasons of the reduction in dimensionality is thus due to the smallness of the vertical scale. Another example of a geometrically constrained system is the flow in a thin soap film. ${ }^{4}$ The geometrical constraint in the ocean and atmosphere is not as pronounced as that in soap films. However, a vertical density gradient and the background rotation of the Earth may further contribute to the 2D behavior of largescale geophysical flows. Laboratory experiments on 2D turbulence are commonly performed in rotating fluids, in

\footnotetext{
${ }^{\text {a) }}$ Also at the Department of Applied Mathematics, University of Twente, The Netherlands.

b) Participates in J. M. Burgers Centre, Research School for Fluid Dynamics, The Netherlands.
}

linearly or two-layer stratified fluids, in electromagnetically driven shallow fluid layers, and, as already mentioned above, in soap films. ${ }^{5}$

Laboratory experiments to improve our understanding of 2D turbulence usually imply rather strong assumptions. For example, in stratified flows, it is often assumed that vertical shear does not interfere too strongly with the planar flow, thus assuming nearly ideal 2D turbulence dynamics. The effect of vertical shear is often parametrized by a linear friction term. ${ }^{6,7}$ Similarly, the effects of Ekman boundary layers in rotating fluids are assumed to be relatively unimportant in the geostrophic interior, where the flow is considered to behave two dimensionally. ${ }^{7}$ Another limitation concerns the presence of lateral (no-slip) domain boundaries. In the laboratory (quasi-)2D turbulence is never unbounded, as is the case in theoretical and numerical (assuming periodic boundary conditions) studies, and it was commonly assumed that these lateral boundaries could be safely ignored. During the past decade, several numerical ${ }^{8-12}$ and experimental ${ }^{13-15}$ studies have been conducted that support the conjecture that under certain circumstances, one should take into account the presence of these lateral boundaries. For example, the quasistationary final states of decaying 2D turbulence critically depend on the shape of the domain (square, rectangular, circular) and the type of boundary conditions. Decaying or forced 2D turbulence in rectangular large-aspect-ratio containers provides another interesting illustration. In both cases, one may observe the emergence of several counterrotating large-scale vortices. One of the most remarkable observations is the spontaneous spin up of a $2 \mathrm{D}$ turbulent flow in square containers with no-slip walls. The turbulent flow, 
initially containing zero net angular momentum (defined with respect to the center of the container), acquires a substantial amount of angular momentum due to strong flowwall interactions. ${ }^{11,16}$

Large-scale flows observed in sea basins and estuaries show similarities with those from laboratory experiments in rectangular containers. A nice example is the satellite image of an array of circulation rings in the Gulf of Aden. ${ }^{17}$ The rings are generated near the mouth of the Gulf of Aden by the northward accelerating Somali Current. The rings with a diameter comparable to the width of the Gulf of Aden then translate westward into the gulf. A similar pattern was observed by Buffoni et al. ${ }^{18}$ and Falco et al. ${ }^{19}$ for the Tyrrhenian Sea and Adriatic Sea, respectively. The energy of these flows is supplied by the wind stress, which exerts a force to the sea surface. The variation in the wind will result in a somewhat random formation of flow structures in these basins. The formation of the array of domain-sized vortices is related to the self-organization of $2 \mathrm{D}$ flows. However, in these geophysical examples, the influence of, for example, the bottom topography and the details of the shape of the sea basin will also contribute to and affect the large-scale flow structures.

Numerical studies of 2D turbulence in square, rectangular, and circular domains are so far hampered by the computational costs. Moreover, the statistical and spectral analysis is not straightforward due to anisotropy and inhomogeneity induced by the lateral boundaries. For example, long-time averaging of confined forced 2D turbulence can only be pursued for relatively small Reynolds numbers [based on the typical root mean square (rms) velocity of the turbulent flow and the domain size]. Today, new techniques are being developed for confined 2D turbulence based on immersed boundary methods, ${ }^{20,21}$ enabling the use of simple computational algorithms together with large-scale parallel computing. We have chosen here to explore yet another welldeveloped and reliable alternative: turbulent flows in a 2D channel geometry by Fourier-Chebyshev pseudospectral methods. Due to the presence of one periodic direction in the channel and homogeneity along lines parallel to the boundaries, both efficient computations can be carried out and statistical and spectral analyses can be conducted rather cleanly.

In this paper, we present results of a numerical study on the dynamics and the dispersion properties of forced 2D turbulence on a periodic-channel domain with no-slip walls. First, we give in Sec. II a description of the problem and the numerical methods used. Then, we investigate the large-scale structure of the flow for different aspect ratios of the domain, see Sec. III. For the largest aspect ratio considered in this study, a set of simulations has been conducted with different integral-scale Reynolds number. The results on the continuously forced flows are then compared to solutions of the Stokes equation for these flow geometries in Sec. IV. Vorticity production at no-slip boundaries might affect the structure and scaling of 2D turbulence and yield modified spectra. These issues will be discussed in Sec. V. Subsequently, the dispersion of a passive tracer is studied, where the tracer is injected continuously at the no-slip walls (see Sec. VI). The role of no-slip walls on enstrophy and tracer variance spectra is discussed in Sec. VII. Finally, in Sec. VIII, we will briefly summarize and discuss the results obtained so far.

\section{PROBLEM DESCRIPTION AND NUMERICAL SETUP}

The 2D flow of an incompressible fluid in the $x, y$ plane, which is described by the velocity field $\mathbf{u}(t, \mathbf{x})=(u, v)$, is governed by conservation of mass and momentum. For a fluid with constant density $\rho$, conservation of mass yields

$$
\nabla \cdot \mathbf{u}=0 .
$$

The Navier-Stokes equation

$$
\frac{\partial \mathbf{u}}{\partial t}+(\mathbf{u} \cdot \nabla) \mathbf{u}=-\frac{1}{\rho} \nabla p+\nu \nabla^{2} \mathbf{u}+\frac{1}{\rho} \mathbf{F}
$$

describes the momentum balance, where $p$ is the pressure, $\nu$ is the kinematic viscosity of the (Newtonian) fluid, and $\mathbf{F}$ is an external force per unit area.

An important alternative variable for describing the flow is the scalar vorticity $\omega$, defined by

$$
\omega=\mathbf{e}_{z} \cdot \nabla \times \mathbf{u}=\frac{\partial v}{\partial x}-\frac{\partial u}{\partial y},
$$

where $\mathbf{e}_{z}$ is the unit vector in the $z$ direction (perpendicular to the plane of flow). Taking the curl of Eq. (2) results in the vorticity equation for the $2 \mathrm{D}$ flow in the $x, y$ plane:

$$
\frac{\partial \omega}{\partial t}+\mathbf{u} \cdot \nabla \omega=\nu \nabla^{2} \omega+\frac{1}{\rho} F_{\omega},
$$

with $F_{\omega}=\mathbf{e}_{z} \cdot \nabla \times \mathbf{F}$. The main difference between the (scalar) vorticity equation (4) and its $3 \mathrm{D}$ vector counterpart is the absence of the term $(\boldsymbol{\omega} \cdot \nabla) \mathbf{u}$, which represents the stretching and tilting of vortex tubes in 3D flows. In 2D unbounded flows, the vorticity equation is actually an advectiondiffusion equation, which expresses that vorticity is merely redistributed-not created (except possibly by an external forcing mechanism).

Consider now the flow domain $\mathcal{D}$ with boundary $\partial \mathcal{D}$ $=\partial \mathcal{D}_{p}+\partial \mathcal{D}_{w}$, with $\partial \mathcal{D}_{p}$ representing the periodic boundary of the channel domain. We demand that the velocity and the vorticity must be periodic at $\partial \mathcal{D}_{p}$. For the lateral side walls, $\partial \mathcal{D}_{w}$, no-slip boundary conditions are imposed, i.e., both the normal and the tangential velocity components must vanish at the wall. Supplemented with an initial condition, the vorticity equation can be integrated to yield the solution at later times. Although the velocity field has to be periodic over the channel length, a constant pressure gradient may be imposed in the longitudinal direction. In our case, the pressure drop over the channel is set to zero.

The equations are numerically solved by using a Fourier-Chebyshev pseudospectral method in the domain $\mathcal{D}$, which is spanned by the rectangle $[0,2 L) \times[-H, H]$, with $H$ the half-width of the channel. The half-width is fixed at $H$ $=1$, while the length of the channel, $2 L$, is varied to obtain aspect ratios $\gamma=L / H=1,2$, and 4 . The no-slip walls of the channel are located at $y= \pm 1$. The velocity and vorticity are expanded in a truncated series of Fourier polynomials for the $x$ direction and in a truncated series of Chebyshev polynomials for the nonperiodic $y$ direction. For the numerical com- 


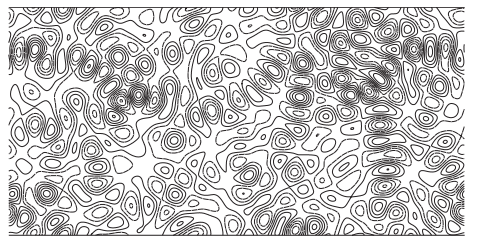

FIG. 1. In physical space, the forcing field $Q_{\omega}$ exhibits a pattern of alternating maxima and minima, with a typical length scale of $2 \pi /\left|\mathbf{k}_{f}\right|$.

putation of the vorticity evolution, a semi-implicit time discretization scheme is applied to the vorticity equation (4), while the nonlinear term in Eq. (4) is treated by the explicit second-order Adams-Bashforth scheme. The diffusion term on the right-hand side of Eq. (4) is then treated by the implicit Crank-Nicolson scheme. Applying the spatial and time discretizations yields a system of equations for the spectral coefficients for the velocity and vorticity which can be resolved by using a specially tailored Gaussian elimination technique. The vorticity values at the walls are not known $a$ priori. Enforcing the vorticity definition (3) with an influence matrix yields the correct boundary values for the vorticity at the walls. ${ }^{22,23}$ A more detailed description of the numerical method and implementation of the influence matrix technique for the simulation of flows in the 2D channel geometry is provided in Ref. 24.

To reach a statistically steady state of the flow, a source of energy is required to balance the loss of energy due to viscous dissipation. For this purpose, the vorticity equation (4) is extended with a time-dependent forcing term. To restrict the vorticity source term $F_{\omega}$ to a certain scale, only Fourier modes with wave vectors $\mathbf{k}_{f}$ within a wave shell $k_{1}$ $\leq\left|\mathbf{k}_{f}\right| \leq k_{2}$ are excited. The precise form of the excitation term $Q_{\omega}$ is

$$
Q_{\omega}(\mathbf{x})=\sum_{k_{1} \leqslant\left|\mathbf{k}_{f}\right| \leqslant k_{2}}\left|A_{\omega}\right| \exp \left(i \theta_{\mathbf{k}}\right) \exp \left(i \mathbf{k}_{f} \cdot \mathbf{x}\right) .
$$

Each mode within the wave shell is excited with the same amplitude $A_{\omega}$ and with a random phase $\theta_{k}$. The forcing field based on a particular excitation $Q_{\omega}$, as illustrated in Fig. 1 for $\gamma=2$, exhibits a pattern of alternating maxima and minima. Due to the random phase at every time level, the position of the maxima and minima can change very rapidly. Although at some locations vorticity is added by the source term at one time, at the next time level, the gained vorticity may be partially canceled by forcing with an opposite contribution. To counteract these (partial) cancellations, the vorticity source term at time $t^{n}$ is correlated to the one at previous times by a Markov chain:

$$
F_{\omega}\left(t^{n}, \mathbf{x}\right)=\left(1-r^{2}\right)^{1 / 2} Q_{\omega}(\mathbf{x})+r F_{\omega}\left(t^{n-1}, \mathbf{x}\right)
$$

where $r$ is the correlation factor. The correlation factor $r$ can be expressed in terms of the correlation time $\tau$ according to

$$
r=\frac{1-\Delta t / 2 \tau}{1+\Delta t / 2 \tau}
$$

Now, the pattern still changes in time, but the changes are more slowly, viz., on the time scale $\tau$. The Markov chain forcing protocol was first used by Lilly ${ }^{25}$ in $2 \mathrm{D}$ turbulence simulations. Later, it was applied in numerical studies of forced 2D turbulence on periodic domains by Maltrud and Vallis $^{26}$ and Oetzel and Vallis. ${ }^{27}$ More recently, this forcing protocol was used by Clercx et al..$^{28}$ and Molenaar et al. ${ }^{29}$ for 2D turbulence simulations in bounded domains with no-slip walls.

The choice of the forcing scale depends on which part of the inertial range, the inverse energy cascade or the direct enstrophy cascade, one wishes to investigate. In the present study, we are interested in the possible role of the no-slip walls as sources of small-scale vorticity, as was suggested by Clercx and van Heijst. ${ }^{16}$ In particular, we plan to study whether the injection of small-scale vorticity can impede the enstrophy cascade. Hence, the flow is forced at large scales (in our case, the wave shell $7 \pi \leq\left|\mathbf{k}_{f}\right| \leq 9 \pi$ ), in order to obtain a clear inertial enstrophy cascade range.

\section{TURBULENCE IN DOMAINS WITH DIFFERENT ASPECT RATIOS}

The aspect-ratio dependence of forced and decaying 2D turbulence in the channel geometry will be first discussed from a phenomenological point of view. The emphasis is put on the emergence of large-scale flow structures, in the form of domain-sized vortical flows. In the next section, a connection will be made with the characteristic Stokes solutions associated with the channel geometry and its aspect ratio.

Table I specifies the settings used for the simulations of forced 2D turbulence in the periodic-channel geometry. The first group of simulations was performed to investigate the influence of the aspect ratio of the computational domain on

TABLE I. The parameters for the forced turbulence simulations: the viscosity $\nu$, the forcing amplitude $A_{\omega}$, the

\begin{tabular}{|c|c|c|c|c|c|c|c|c|}
\hline Run No. & $\gamma$ & $\nu \times 10^{-4}$ & $A_{\omega}$ & $\tau \times 10^{-2}$ & $k_{f}$ & $K$ & $M$ & $\Delta t$ \\
\hline $1 \mathrm{~A} 1$ & 1 & 5 & 4 & 1 & {$[7 \pi, 9 \pi]$} & 256 & 256 & $5 \times 10^{-4}$ \\
\hline $1 \mathrm{~A} 2$ & 2 & 5 & 4 & 1 & {$[7 \pi, 9 \pi]$} & 512 & 256 & $5 \times 10^{-4}$ \\
\hline $1 \mathrm{~A} 4$ & 4 & 5 & 4 & 1 & {$[7 \pi, 9 \pi]$} & 1024 & 256 & $5 \times 10^{-4}$ \\
\hline $2 \mathrm{~A} 4$ & 4 & 5 & 8 & 1 & {$[7 \pi, 9 \pi]$} & 1024 & 256 & $2.5 \times 10^{-4}$ \\
\hline $3 \mathrm{~A} 4$ & 4 & 2 & 8 & 1 & {$[7 \pi, 9 \pi]$} & 1280 & 320 & $8 \times 10^{-5}$ \\
\hline $4 \mathrm{~A} 4$ & 4 & 1 & 8 & 1 & {$[7 \pi, 9 \pi]$} & 2048 & 512 & $4 \times 10^{-5}$ \\
\hline
\end{tabular}
correlation time $\tau$, and the forcing range. The spatial resolution used to resolve the flow is given by the number of Fourier and Chebyshev polynomials, $K$ and $M$, respectively. Finally, $\Delta t$ is the time step. 

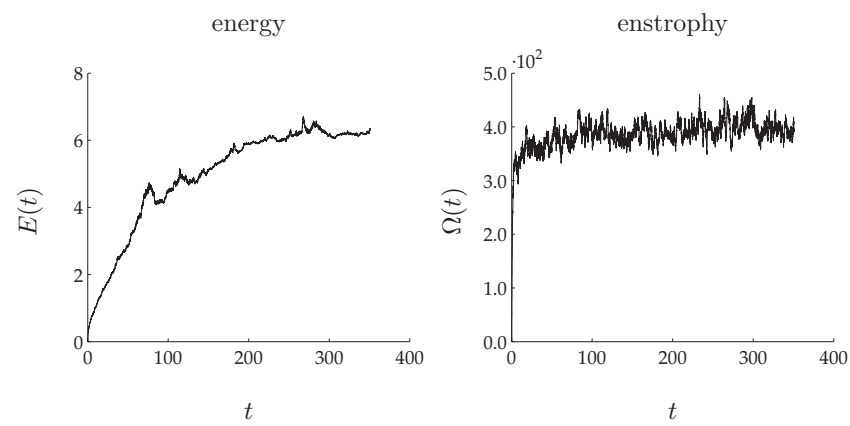

FIG. 2. Energy and enstrophy evolution are displayed for the simulation of forced turbulence on a periodic channel with aspect ratio $\gamma=4$. The viscosity is equal to $\nu=5 \times 10^{-4}$, yielding a Reynolds number of $\operatorname{Re} \approx 1800$ when the energy reaches a constant level.

the formation of domain-sized vortices and the number of these structures appearing in the channel. The amplitude of the vorticity source term is then fixed to a value of $A_{\omega}=4$ [see Eq. (5)] and the forcing correlation time is fixed at $\tau$ $=1 \times 10^{-2}$. The correlation time is chosen to be small in order to have a fast changing forcing field, but it is still at least 20 times larger than the used time step and hence well resolved. The viscosity parameter is also fixed to one value, viz., $\nu=5 \times 10^{-4}$. The grid resolution limits the maximum value for the viscosity as the boundary-layer thickness has to be resolved. The actual Reynolds number is based on the half-width of the channel, $H=1$, and the rms velocity $U_{\text {rms }}$, thus, $\operatorname{Re}=U_{\text {rms }} H / \nu=U_{\text {rms }} / \nu$. The rms velocity is calculated from the kinetic energy once the turbulence has reached a statistically steady state.

Figure 2 gives a graphical representation of the evolution of the kinetic energy and the enstrophy for a domain with aspect ratio $\gamma=4$. The initial condition is a zero vorticity field, i.e., the flow is initially at rest. Hence, the energy is starting at zero but increases as energy is injected by the forcing. For $t>250$, the energy is seen to fluctuate around a constant level, and the mean energy injection by the forcing is thus canceled by the mean viscous dissipation. The values of the kinetic energy and enstrophy during the statistically steady state are specified in Table II. For $\gamma=1$ and $\gamma=2$, there are stronger fluctuations in the energy and it is less clear whether the mean energy has reached the constant level. To estimate the advection time scale in the simulations, the integral-scale eddy turnover time is calculated according to

TABLE II. The mean value of the kinetic energy $E$ and the enstrophy $\Omega$ during the statistically steady state. The Reynolds number Re, the approximate rms velocity $U_{\mathrm{rms}}$, and the eddy turnover time $t_{e}$ according to the energy level in the same stage.

\begin{tabular}{lccccc}
\hline \hline Run No. & $\operatorname{Re}$ & $E$ & $\Omega$ & $U_{\text {rms }}$ & $t_{e}$ \\
\hline $1 \mathrm{~A} 1$ & 1300 & 0.8 & $2.8 \times 10^{1}$ & 0.65 & 1.5 \\
$1 \mathrm{~A} 2$ & 1900 & 3.6 & $2.0 \times 10^{2}$ & 0.95 & 1.1 \\
$1 \mathrm{~A} 4$ & 1800 & 6.5 & $4.0 \times 10^{2}$ & 0.9 & 1.1 \\
& & & & & \\
$2 \mathrm{~A} 4$ & 3800 & 28 & $1.6 \times 10^{3}$ & 1.9 & 0.54 \\
$3 \mathrm{~A} 4$ & 10000 & 32 & $2.7 \times 10^{3}$ & 2.0 & 0.50 \\
$4 \mathrm{~A} 4$ & 20000 & 36 & $4.1 \times 10^{3}$ & 2.0 & 0.50 \\
\hline \hline
\end{tabular}

(a) stream function
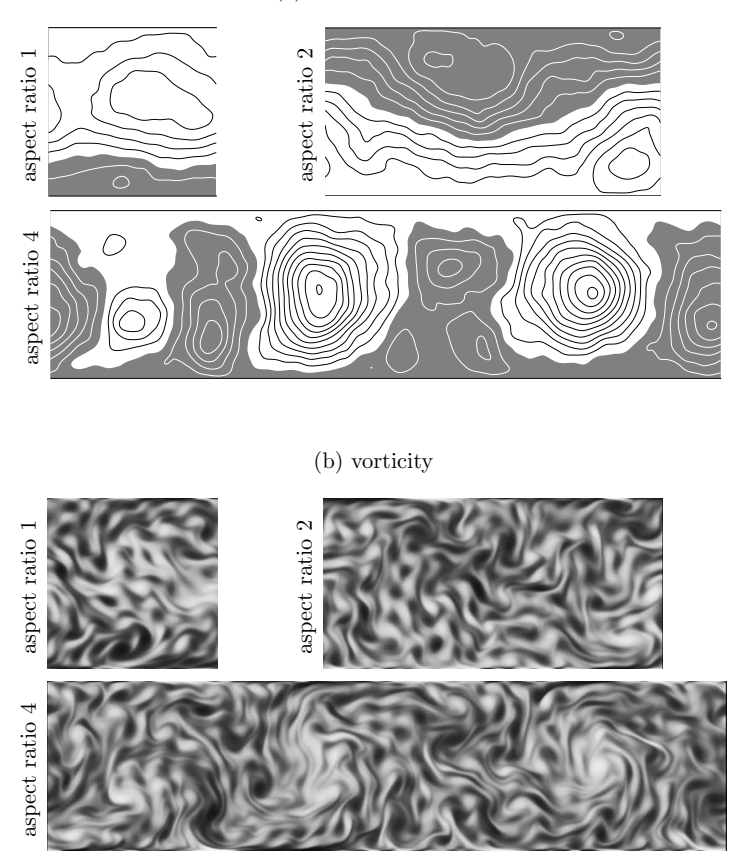

FIG. 3. Stream-function contour plots (a) and vorticity fields (b) of the forced turbulence simulations on a rectangular domain with aspect ratios $\gamma=1,2$, and 4 . Regions where the stream function is positive and negative are colored white and gray, respectively. The maximum positive and negative vorticity values are colored white and black, respectively.

$t_{e}=H / U_{\mathrm{rms}}=1 / U_{\mathrm{rms}}$. Note that the enstrophy reaches a constant level more rapidly than the energy. This indicates that the length scale $l_{E}=(E / \Omega)^{1 / 2}$, which is associated with the size of the energy-containing eddies of the flow, is slowly increasing.

Figure 3 shows snapshots of the stream function for different aspect ratios $\gamma$ of the domain. In all the cases, a statistically steady state is reached. For aspect ratios $\gamma=1$ and 2 a strong quasiunidirectional flow develops. Recall that there is no pressure drop imposed over the channel length, so the unidirectional flow spontaneously develops. This unidirectional flow can be in either direction, as was indeed found by performing an ensemble of simulations for $\gamma=1$ (Fig. 4). The direction of the volume flow $u_{V}(t)=\int u(t, \mathbf{x}) d y$ is fixed over a long period of time. The channel flow shows strong fluctuations in $u_{V}$ and at certain times, $u_{V}$ changes sign. The forcing

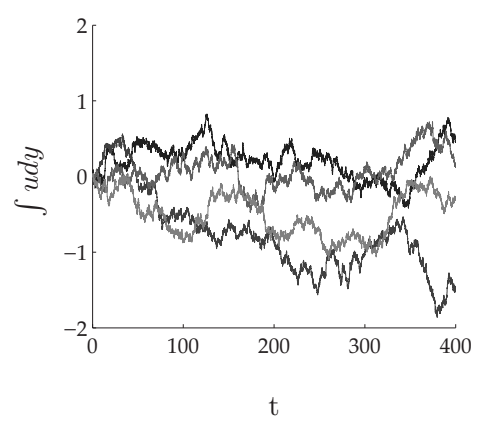

FIG. 4. The time evolution of the volume flow, $u_{V}(t)=\int u(t, \mathbf{x}) d y$, for four individual simulations in a channel with aspect ratio $\gamma=1$. As the flow is divergence-free, the volume flow $u_{V}$ does not depend on $x$. The viscosity and forcing amplitude are the same for all four runs (1A1). 
correlation time is $\tau=0.01$ and is four orders of magnitude than the average time between two subsequent sign reversals of $u_{V}$. Earlier simulations of forced and decaying 2D turbulence on a bounded square domain have revealed a similar phenomenon, known as "spontaneous spin up," with the bulk of the fluid rotating in one direction, either clockwise or anticlockwise. ${ }^{12,15,29}$ For an aspect ratio of $\gamma=1$, the strong unidirectional flow is slightly perturbed by a one domainsized circulation cell, as is clearly visible in the streamfunction contour plot, see Fig. 3. In the case $\gamma=2$, we observe two of these cells, one with clockwise rotation and the other with anticlockwise rotation, thus perturbing the alongchannel flow (see Fig. 3). For an aspect ratio $\gamma=4$, we observe four to six circulation cells, and an along-channel flow can be hardly observed. The presence of this along-channel flow for the smaller aspect ratios seems to be a direct consequence of the presence of the periodic boundary conditions. The influence of these boundaries is reduced for largeaspect-ratio channels, which-together with the isotropic forcing - apparently supports the formation of the circulation cells. In large-aspect-ratio domains, the flow will likely organize into a larger number of circulation cells. As a consequence of the periodic boundary conditions, the number of cell will always be even. A simulation for a domain with $\gamma$ $=3$ revealed four elliptic cells. This in contrast to the case with $\gamma=4$, where the cells are more circular. In laboratory experiments, a pattern of domain-sized cells is typically observed, while the presence of solid boundaries does not allow the formation of a unidirectional flow. Therefore, further simulations will be restricted to domains with an aspect ratio $\gamma=4$.

The array of vortices that is observed for a periodic channel with aspect ratio $\gamma=4$ resembles the results for the spin-up experiments in rectangular containers by van Heijst et $a .^{30}$ and van de Konijnenberg et al.,${ }^{31}$ as well as the experiments conducted in a stratified fluid by Maassen et al. ${ }^{15}$ It should be mentioned, however, that these latter laboratory experiments concerned decaying quasi-2D flows.

The organization of the flow into domain-sized cell structures is less clear from snapshots of the vorticity field [see Fig. 3(b)]. In the domain-sized regions where the stream function is positive, the vorticity field is characterized by smaller-scale vorticity patches that are predominantly positive and vice versa. The emergence of small-scale vorticity patches is related to the forcing mechanisms itself and the interaction of the turbulent flow with the lateral no-slip walls. The latter effect is clearly illustrated by the detachment of thin boundary layers containing high-amplitude vorticity and its subsequent roll-up, leading to small-scale vortices.

In the second set of simulations, the aim is to investigate the impact of increased Re values. For these simulations, the aspect ratio is set to $\gamma=4$. In the first simulation of this set (2A4, see Table I), the final vorticity field of the simulation with $\operatorname{Re} \approx 1800$ and $\gamma=4$ (simulation 1A4) is used as initial data and we doubled the forcing amplitude $\left(A_{\omega}=8\right)$. After $t=100$, the flow reaches again a statistically steady state but with an increased kinetic energy of $E \approx 28$ (see Table II). The Reynolds number has thus increased from $\operatorname{Re} \approx 1800$ to
3800. To increase the Reynolds number even further, we performed a simulation for which the viscosity was decreased while keeping the forcing amplitude fixed at $A_{\omega}=8$. The vorticity field of the previous simulation is again used to provide the initial data, and the spatial resolution is increased to resolve the smaller flow structures. Thereafter, the Reynolds number is increased even further by reducing the viscosity once more. In the latter two cases, the statistically steady state is not completely. The energy input by the forcing is then not balanced by the viscous dissipation. This yields an energy increase of approximately $1 \%$ per eddy turnover time.

In Fig. 5, vorticity snapshots of a few simulations are displayed; the Reynolds number is varied between $\operatorname{Re} \approx 1800$ and 20000 . The snapshots taken at low Re values reveal many vorticity structures with length scales comparable to the forcing scale. Vorticity patches (or filaments) with smaller scales are quickly removed by viscous dissipation. If the Reynolds number is increased, the spatial structure of the forcing is less persistently present in the vorticity field, and small-scale vortices and vorticity filaments start to emerge more prominently. These latter vorticity structures predominantly originate from the walls and they survive more easily because viscous dissipation is now acting on substantially smaller scales. Note that the vortices also become more compact, i.e., they have a smaller size with a larger vorticity amplitude (see also Wells et $a l .^{32}$ ). Moreover, some vorticity patches that have detached from the wall are advected by and stretched between the domain-sized circulation cells. This typically leads to vorticity filaments that are aligned perpendicular to the wall.

\section{ARE LARGE-SCALE STRUCTURES RELATED TO STOKES MODES?}

For decaying turbulence, the long-time behavior is characterized by a steadily decreasing Reynolds number. Eventually, the flow ceases to be turbulent and the fully laminar regime is obtained (and any nonlinearity is depleted). In the limit of $\operatorname{Re} \rightarrow 0$, the solution is described by the Stokes equation, i.e., the advection term in the vorticity equation (4) can be neglected,

$$
\frac{\partial \omega}{\partial t}=\nu \nabla^{2} \omega \text { in } \mathcal{D}
$$

To investigate the resemblance between the domain-sized structures observed in forced and decaying 2D turbulence and the Stokes modes of a confined viscous flow, we now discuss the Stokes modes in a periodic-channel domain.

Solutions of the Stokes equation vary for different geometries of the domain and can be found by separation of variables, i.e., by writing the solution as $\omega(\mathbf{x}, t)=T(t) \bar{\omega}(\mathbf{x})$. The time-dependent part is then given by $T(t)=\exp (\nu \sigma t)$, where $\sigma$ is a constant yet to be determined. The spatial part of the solutions, $\bar{\omega}(\mathbf{x})$, is governed by

$$
\nabla^{2} \bar{\omega}-\sigma \bar{\omega}=0 \quad \text { in } \mathcal{D} .
$$

The spatial solution depends on the applied boundary conditions, see, for instance, the work of Orszag et al. ${ }^{33}$ for a periodic-channel domain and van de Konijnenberg et al. ${ }^{34}$ 


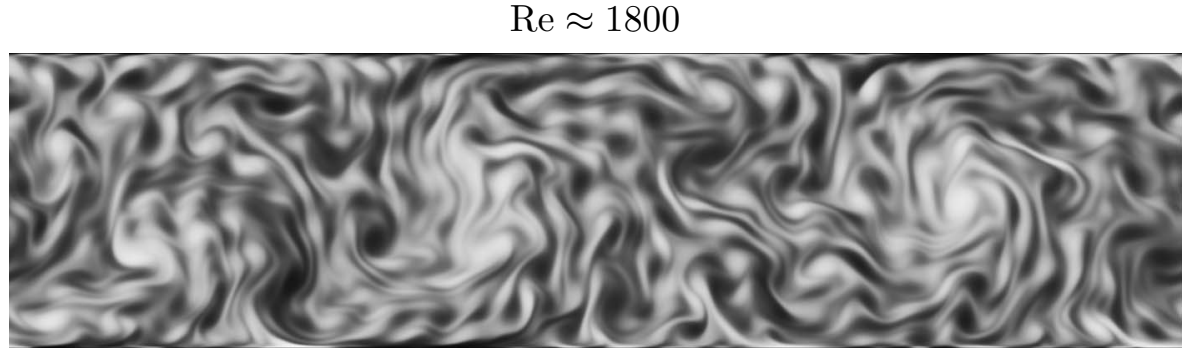

$\mathrm{Re} \approx 3800$

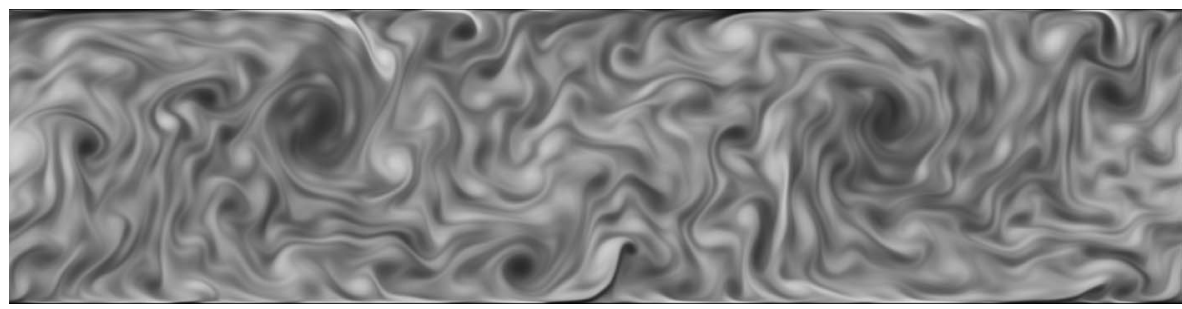

$\mathrm{Re} \approx 10000$

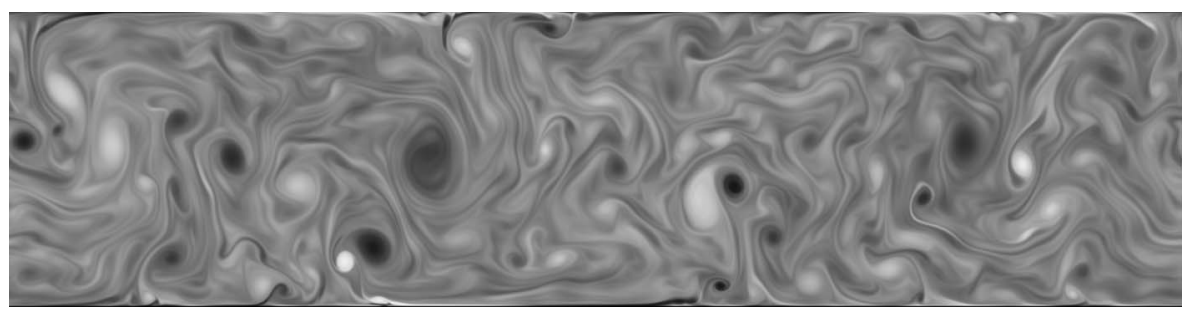

$\mathrm{Re} \approx 20000$

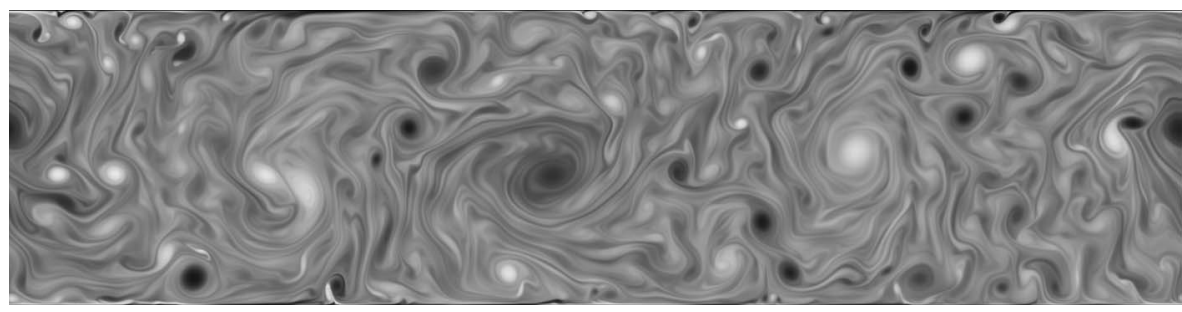

FIG. 5. Results of simulations of forced turbulence in a channel domain with aspect ratio $\gamma=4$ : typical vorticity snapshots for $\operatorname{Re} \approx 1800,3800$, 10000 , and 20000. for the bounded square domain. In the case of the 2D periodic channel, we assume for the $x$ direction that the solution can be written as a Fourier series. Equation (9) can then be reformulated as

$$
\frac{\partial^{2} \bar{\omega}}{\partial y^{2}}+\mu^{2} \bar{\omega}=0,
$$

where we have introduced $\mu^{2}=-\sigma-k^{2}$, with $k$ the wave number of the Fourier polynomial. Symmetric solutions are then given by

$$
\bar{\omega}(\mathbf{x})=-A\left(\mu^{2}+k^{2}\right) \cos (\mu y) \exp (i k x)
$$

and the antisymmetric solutions by

$$
\bar{\omega}(\mathbf{x})=-A\left(\mu^{2}+k^{2}\right) \sin (\mu y) \exp (i k x) .
$$

Here, $A$ is a complex constant that determines the phase of the Fourier polynomial. Without loss of generality, we assume that the no-slip walls are located at $y= \pm 1$. For illustration purposes, we now choose a domain with aspect ratio $\gamma=2$; thus, $\mathcal{D}=[0,4) \times[-1,1]$. The channel length, which is equal to $2 L$, prescribes the possible wave numbers $k$ in the $x$ direction. For instance, $k=\pi / L$ is the gravest nonzero wave number. For a given $k$, the value for $\mu$ is determined by the boundary conditions and by demanding that the velocity field is divergence free. More details of the possible Stokes solutions and values for $k$ and $\mu$ are given in the Appendix. Any combination of $k$ and $\mu$ results in a negative $\sigma$, which is required for the time-dependent part to remain finite (and decaying) in time. The gravest mode with the smallest $k$ and $\mu$ decays at the slowest rate. Only the asymmetric modes with $k=0$ contain net momentum in the $x$ direction. Both in simulations on decaying $2 \mathrm{D}$ turbulence ${ }^{11,15}$ and in experiments on stratified or rotating decaying turbulence ${ }^{15,30,34}$ the gravest Stokes mode emerges for the long-time limit in square containers. In Fig. 6(b), the gravest Stokes mode for $k=\pi / 2$ is given on a domain with aspect ratio $\gamma=2$. The solution consists of two counter-rotating cells with a size comparable to the width of the periodic channel. The gravest mode, i.e., the antisymmetric mode with $k=0$ and $\mu=\pi / 2$ [Fig. 6(d)] is the slowest decaying Stokes mode. Its appear- 


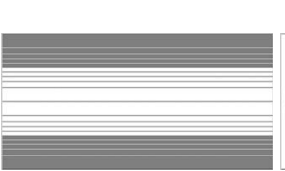

(a) $k=0, \mu=\pi$

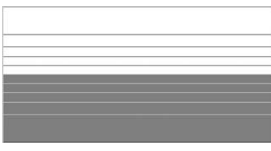

(d) $k=0, \mu=\pi / 2$

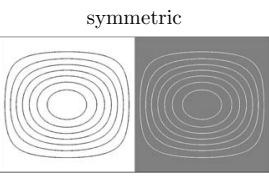

(b) $k=\pi / 2, \mu=2.64$ antisymmetric

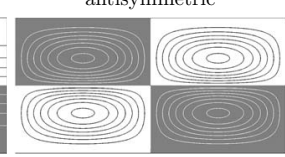

(e) $k=\pi / 2, \mu=4.34$

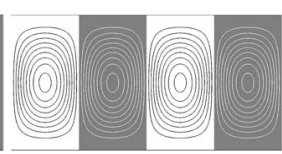

(c) $k=\pi, \mu=2.18$

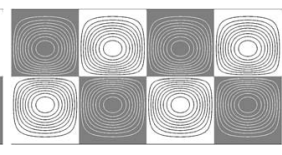

(f) $k=\pi, \mu=4.05$
FIG. 6. The stream function of the gravest symmetric and antisymmetric solutions to the Stokes equation on a periodic-channel domain with $\gamma=2$ for $k=0$ [(a) and (d)], $k=\pi / 2[(\mathrm{~b})$ and (e)], and $k=\pi[(\mathrm{c})$ and (f)]. White and gray areas denote positive and negative values of the stream function, respectively.

ance closely resembles a Poiseuille flow. The velocity profile is not parabolic but has a cosine profile, $\bar{u}=(\pi / 2) \cos (\pi y / 2)$. The difference with the Poiseuille flow is the absence of a pressure drop associated with the velocity profile-for the Poiseuille flow a pressure gradient is essentially required to sustain the flow.

The relation of the Stokes modes to the self-organization of (forced) 2D turbulence is uncertain. Note that the Stokes modes form a complete basis on which the flow field can be projected. ${ }^{35}$ Each individual mode is divergence-free and satisfies the boundary conditions. Moreover, the spatial structure is independent of the Reynolds number, while the viscous decay of the mode is governed by an exponentially decreasing amplitude. The minimum enstrophy states, which can be obtained by variational calculus, are identical to the spatial structure of the Stokes modes. ${ }^{36}$ One should realize that the same can be said about Fourier modes for periodic domains. Each Fourier mode itself is a solution of the Stokes equation, with the amplitude exponentially decreasing. The nonlinear interactions in the Navier-Stokes equations are governed by the triad interactions between modes with different wavelengths. For forced turbulence in a doubleperiodic domain, these nonlinear interactions drive the inverse cascade, which leads to a condensation of energy in the smallest accessible wave number. ${ }^{37}$ In the periodic-channel domain, the nonlinear term will result in interactions between the different Stokes modes. Whether in this case the energy condenses on the gravest Stokes mode is an interesting open question.

To investigate the largest scales in the flow, we analyzed the stream function. In the stream function, the largest flow structures are most clear, while in the vorticity field, smaller scales become more pronounced. The stream function is decomposed into Fourier polynomials for the periodic $x$ direction, while no decomposition is applied for the $y$ direction. Each Fourier component of the stream function is denoted by (the real part of) $\varphi_{k}(y) \exp (i k x)$ with $k$ the wave number. The complex amplitude $\varphi_{k}(y)$ includes that the amplitude and the phase of each Fourier polynomial might change along $y$. Note that the spatial structure of the Stokes modes can be (a) $k=0$

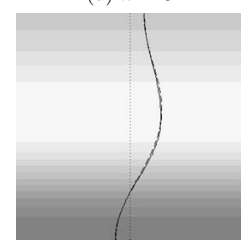

(b) $k=\pi$

(c) $k=2 \pi$

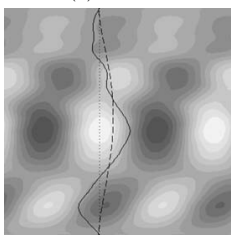

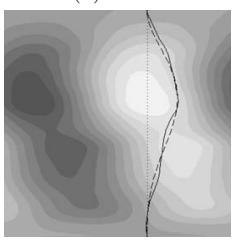

(d) $k=0$

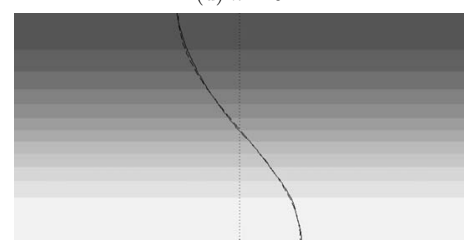

(e) $k=\pi / 2$

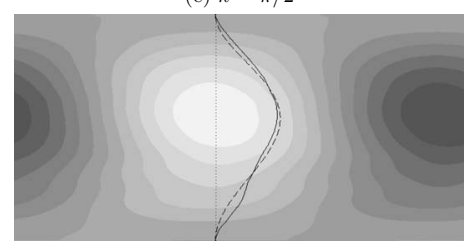

(f) $k=\pi$

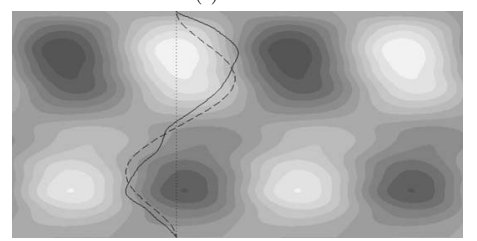

FIG. 7. A snapshot of the stream function is decomposed into the lowest $k$ wave numbers for aspect ratios $\gamma=1[(\mathrm{a})-(\mathrm{c})]$ and $\gamma=2[(\mathrm{~d})-(\mathrm{f})]$ for simulations 1A1 and 1A2. The cross section of the stream function (drawn) is compared to the profile of the gravest Stokes modes (dashed). This profile is constructed from the gravest even and odd Stokes mode for given $k$. The amplitude of the stream-function components is 0.39 (a), 0.11 (b), and 0.04 (c) for $\gamma=1$ and 0.49 (d), 0.32 (e), and 0.08 (f) for $\gamma=2$.

written in the same form, but then the phase is by definition independent of $y$.

In Fig. 7, we investigate whether the $k$ component of the stream function can be constructed using only the two gravest Stokes modes (i.e., the two modes with the lowest valued $\mu$ for given $k$ ). The gravest modes for various $k$ are given in Fig. 6. The profile of the Stokes modes is compared to the cross section of $\varphi_{k}(y) \exp (i k x)$ in Fig. 7. For the aspect ratio $\gamma=1$, the $k=0$ component has the largest contribution to the total stream function. The amplitude of this Fourier component is $\left|\varphi_{k}(y)\right|=0.39$, while it is 0.11 for the $k=\pi$ component. This agrees with the strong unidirectional flow observed. The $k=0$ component of the stream function is described well by the gravest antisymmetric and symmetric Stokes modes. For the aspect ratio $\gamma=2$, the amplitude of the $k=\pi / 2$ component of the stream function is of the same order as the $k=0$ component $\left[\left|\varphi_{k}(y)\right|=0.32\right.$ versus 0.49 , respectively]. In the flow, this relates to the presence of two domain-sized circulation cells on top of the unidirectional flow. The results for $\gamma=2$ seems to indicate that isotropic modes, which are characterized by predominantly circular cells [viz. Figs. 6(b) and 6(f)], are favored over lower-order anisotropic modes [e.g., Figs. 6(c) and 6(d)]. For instance, in the $k=\pi$ component, the antisymmetric mode $(A=0.065)$ is an order of magnitude larger than the lower-order symmetric mode $(A=0.008)$. The former of the two has a more isotropic character. For the domain with the aspect ratio $\gamma=4$, most energy is present in the $k=\pi / 2$ component of the flow (Fig. 8), which explains the presence of four strong circulation cells for $\gamma=4$. This is also visible in the energy spectra that we will discuss in the next section. The $k=0$ component is much weaker and for 
(a) $k=0$

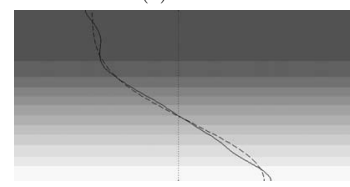

(b) $k=\pi / 4$

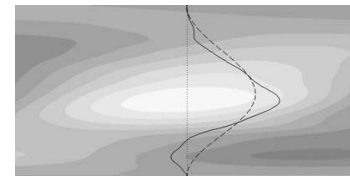

(c) $k=\pi / 2$

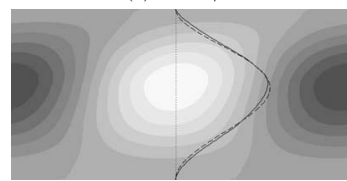

(d) $k=0$

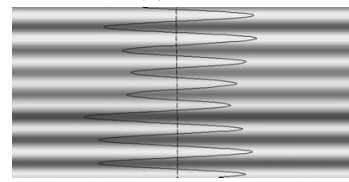

(e) $k=\pi / 4$

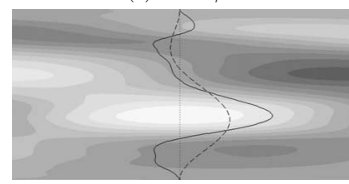

(f) $k=\pi / 2$

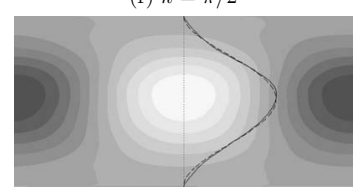

FIG. 8. A snapshot of the stream function is decomposed into the lowest $k$ modes for an aspect ratio of 4 with $\operatorname{Re} \approx 1800$ [(a)-(c)] and 20000 [(d)-(f)]. Only half a channel length is shown. The cross section of the stream function (drawn) is compared to the profile of the gravest Stokes modes (dashed). This profile is constructed from the gravest even and odd Stokes mode for given $k$. The amplitude of the stream-function components is 0.51 (a), 0.19 (b), and 1.39 (c) for $1 \mathrm{~A} 4$ and 0.04 (d), 0.17 (e), and 1.5 (f) for 4A4.

the highest Reynolds number, we only retrieve the $k=0$ component of the forcing field. In this case, the unidirectional flow is very weak and the gravest Stokes modes with $k=0$ are not visible. The $k=\pi / 2$ component of the stream function is described well by the symmetric lowest-order Stokes mode. The flow dynamics seems to favor this isotropic mode above anisotropic modes with lower wave numbers $(k=0$ and $k=\pi / 4$ ). For further research, it could be interesting to investigate the stability of isolated Stokes modes at large Reynolds numbers. Especially, the stability of the unidirectional flow (the gravest $k=0$ mode) for different aspect ratios could give more insight why this mode is practically absent for $\gamma=4$.

\section{SPECTRAL DYNAMICS OF WALL-BOUNDED TURBULENCE}

For 2D turbulence, Kraichnan ${ }^{1}$ formulated the idea of a dual cascade, an inverse cascade of energy to large scales, and a direct cascade of enstrophy to small scales. ${ }^{1}$ The inverse energy cascade and the related $k^{-5 / 3}$ scaling of the energy spectrum has been confirmed by numerical simulations, see, e.g., Refs. 25 and 38-40, and by laboratory experiments, see, e.g., Refs. 41 and 42. The direct enstrophy cascade and its $E(k) \sim k^{-3}$ scaling is subjected to more debate from both the theoretical and the observational side. The $k^{-3}$ scaling is on the verge of breaking the locality of the energy transfer. A large problem in validating the $k^{-3}$ scaling in the enstrophy cascade range is that the Reynolds number must be large to obtain a scale separation between the energy injection scale and the dissipation scale. To restrict the dissipation to larger wave numbers, one often resorts to the use of hyperviscosity, i.e., the viscous term in the Navier-Stokes equation is replaced by a similar term with a higher-order version of the Laplace operator. However, one needs additional boundary (a) interior $(y=0)$

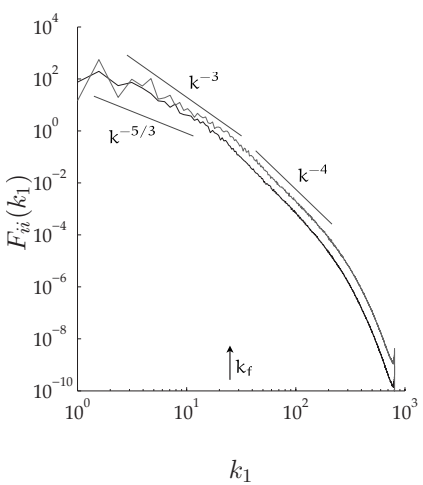

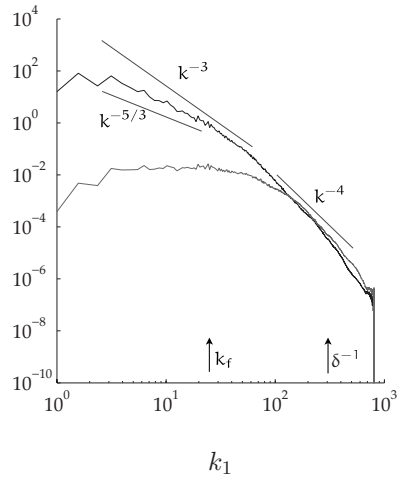

(b) wall region $(y= \pm 0.99)$

FIG. 9. Longitudinal (black) and transverse (gray) specta along a line parallel to the wall (a) in the interior $(y=0)$ and close to the wall (averaged over $y= \pm 0.99)(\mathrm{b})$

conditions to complement the no-slip conditions. In our aim to investigate the importance of no-slip walls, we will restrict ourselves to regular viscosity. Using hyperviscosity can give additional insights but this is left for a later study.

No-slip boundaries convert large-scale energy into small-scale energy. For decaying turbulence Clercx and van Heijst ${ }^{16}$ observed a $-\frac{5}{3}$ slope in the energy spectrum measured near the wall, ranging from small scales related to the boundary-layer thickness toward large scales. This suggests that there is an inverse cascade of energy in this range (and no direct enstrophy cascade). The same phenomenon is observed in simulations and laboratory experiments of rotating fluids by Wells et al. ${ }^{32}$ In these experiments, the background rotation is modulated, causing a constant creation and injection of vorticity structures at the wall. Simulations in a square domain with no-slip boundaries were performed by Molenaar et al. ${ }^{29}$ who applied large-scale forcing. However, in these simulations, no clear $-\frac{5}{3}$ spectral slope was observed near the wall. This might be due to either the moderate Reynolds number used or the presence of the forcing.

In this section, we focus on the spectral dynamics of the turbulent velocity field close to solid boundaries, with emphasis on the enstrophy cascade. For the computation of the one-dimensional longitudinal and transverse spectra, we use the Fourier components $\widetilde{\mathbf{u}}\left(k_{x}, y\right)$ of the velocity field. The longitudinal spectrum is defined as $F_{11}\left(k_{x}, y\right)=\frac{1}{2}\left|u\left(k_{x}, y\right)\right|^{2}$ and the transverse spectrum as $F_{22}\left(k_{x}, y\right)=\frac{1}{2}\left|v\left(k_{x}, y\right)\right|^{2}$. To obtain a wide inertial range, the results of simulation 4A4 (see Table I) for the highest Reynolds number are used. The spectra are averaged over five integral-scale eddy turnover times. In Fig. 9, we plot the longitudinal and transverse spectra, which were calculated along a line in the interior $(y=0)$ and along a line near the wall averaged over both $y=+0.99$ and $y=-0.99$.

In the interior, the spectrum is strongly affected by viscous dissipation, and hence, the spectrum decays very strongly. The Kolmogorov wave number defined by $k_{d} \approx\left(\eta / \nu^{3}\right)^{1 / 6}$ is related to the rate of enstrophy dissipation $\eta$. An approximate local measure of $\eta$ is given by 


$$
\eta_{l}=\nu|\nabla \omega|^{2},
$$

which gives the value $\eta_{l} \approx 40$ in the interior of the domain. This yields for the local Kolmogorov wave number a value of $k_{d} \approx 200$. Due to the strong dissipation, there is no clear power-law scaling in the enstrophy cascade range. Nevertheless, a small power-law range can be observed with a slope steeper than $k^{-3}$. The range of the inverse energy cascade, i.e., $k<k_{f}$, is too short to observe any significant $k^{-5 / 3}$ slope. Note, however, that there is no condensation of energy in a single wave number as was observed in a double-periodic domain. Most of the energy is located in the Fourier component with $k=\pi / 2$. This is in agreement with our findings in the previous section that the symmetric Stokes mode with $k=\pi / 2$ is dominating the stream function.

Near the wall, the spectra reveal a completely different picture. The most striking difference is that there is more energy located at larger wave numbers compared to the energy spectrum in the interior. This seems to be in agreement with the idea that injection of boundary vorticity acts as a forcing mechanism, as was suggested in Ref. 16. The boundary-layer thickness $\delta$ can be estimated by using

$$
\delta^{2}=\frac{\int_{\partial \mathcal{D}}(\partial u / \partial y)^{2} d s}{\int_{\partial \mathcal{D}}(\partial \omega / \partial y)^{2} d s},
$$

which yields $\delta \approx 3 \times 10^{-3}$ when averaged over both boundaries and over five different vorticity snapshots. For wave numbers $k \leqq \delta^{-1} \approx 300$, the longitudinal spectrum does not reveal a range with a clear $-\frac{5}{3}$ slope, as was reported in Refs. 16 and 32. If there is injection of vorticity at scales proportional to $\delta$, it is probably overcast by the energy input due to the forcing. However, the enstrophy is much larger near the wall than in the interior. The local enstrophy dissipation, measured by Eq. (13), here has a value $\eta_{l} \approx 6 \times 10^{3}$, thus, the local Kolmogorov wave number is then $k_{d} \approx 400$, which is in the same order as the wave number related to the boundarylayer thickness. The transverse spectrum becomes flat for wave numbers $k \lesssim 10^{2}$, which is related to the distance between the wall and the line along which the spectra are determined.

\section{DISPERSION OF A TRACER INJECTED AT THE WALL}

The transport of a passive tracer, $c(\mathbf{x}, t)$, is governed by the advection-diffusion equation,

$$
\frac{\partial c}{\partial t}+\mathbf{u} \cdot \nabla c=\kappa \nabla^{2} c
$$

with $\kappa$ the diffusion coefficient of the tracer. The relative importance of advective and diffusive transport of a passive tracer is indicated by the Péclet number, $\mathrm{Pe}=U_{\mathrm{rms}} H / \kappa$. The ratio between the Péclet and Reynolds number is the Schmidt number, $\mathrm{Sc}=\mathrm{Pe} / \mathrm{Re}=\nu / \kappa$.

Here, we are particularly concerned with the dispersion of a passive tracer that is continuously injected at the wall. Therefore, an influx of tracer material is specified at the upper wall,
TABLE III. The parameters for the tracer dispersion simulations in forced turbulence: the tracer diffusivity $\kappa$ and the Schmidt number Sc. The spatial and time resolution is specified by the number of Fourier polynomials $K$, the number of Chebyshev polynomials $M$, and the time step $\Delta t$.

\begin{tabular}{lcccc}
\hline \hline$\kappa \times 10^{-4}$ & Sc & $K$ & $M$ & $\Delta t \times 10^{-5}$ \\
\hline 0.5 & $\frac{1}{4}$ & 1280 & 320 & 8 \\
2.0 & 1 & 1280 & 320 & 8 \\
4.0 & 2 & 1536 & 384 & 8 \\
\hline \hline
\end{tabular}

$$
\left.\kappa \frac{\partial c}{\partial n}\right|_{y=1}=1,
$$

and a negative influx at the lower wall,

$$
\left.\kappa \frac{\partial c}{\partial n}\right|_{y=-1}=-1 .
$$

Although tracer diffusion is very slow for high Péclet numbers, the mixing rate of the tracer can be increased by the turbulent velocity field. In strain dominated regions of the velocity field, patches of tracer material are stretched into long filaments, thus enhancing tracer gradients, leading to a faster tracer diffusion.

We performed three simulations in which a tracer material was injected at the wall. In Table III, the resolutions and other parameters that are used for the simulations are specified. The forced turbulent velocity field, which is taken from simulation 3A4, is identical for all three simulations and has a typical Reynolds number of $\mathrm{Re} \approx 10000$. The values for the tracer diffusion coefficient are varied to obtain results for $\mathrm{Sc}=\frac{1}{4}, 1$, and 2. For the latter case, the resolution used to solve the tracer concentration is increased in order to resolve the thin tracer filaments. The time step is taken constant in all simulations to keep the vorticity forcing, and consequently the flow evolution, the same for all three simulations.

Figure 10 shows the tracer distribution after approximately 40 integral-scale eddy turnover times after the injection was started. For all the Schmidt numbers, the dispersion of tracer material is roughly similar. The main difference is that tracer gradients are smoothed more quickly for smaller Péclet numbers. Most pronounced are the bursts of tracer material between the domain-sized vortices. For the situation of four well defined domain-sized circulation cells, a schematic representation is given in Fig. 11. Between one vortex with positive circulation and one vortex with negative circulation (from left to right), tracer material originating from the lower wall is advected into the domain and crosses the channel. Tracer material originating from the upper wall is mainly advected into the area between a negative and a positive vortex (once again, from left to right).

In case the circulation cell consists of one single vortex, which is basically fed by the global forcing, hardly any tracer material enters this region. This tracer material is usually contained in small vortices originating from the wall region as a result of the roll-up of the thin boundary layers. The tracer material can enter the strong vortex by two mechanisms: by diffusion and by (partial) merger of this dominant vortex with a tracer containing vortex. The first mechanism 
vorticity field, $\operatorname{Re} \approx 10000$

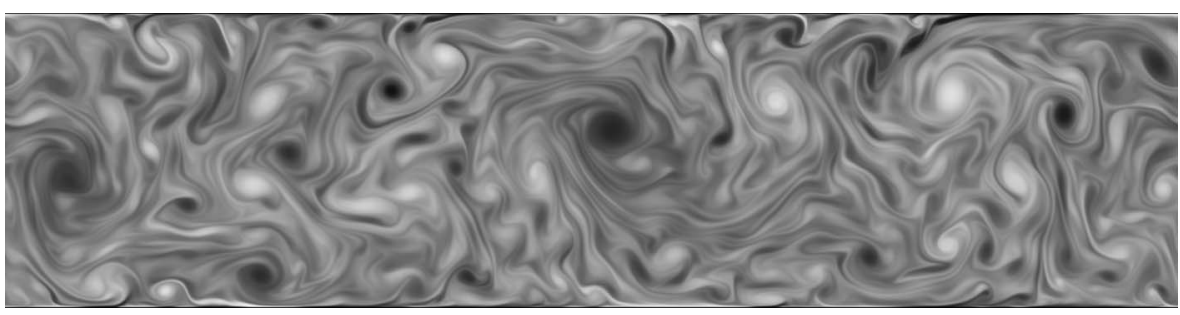

tracer field $\mathrm{Sc}=1 / 4$

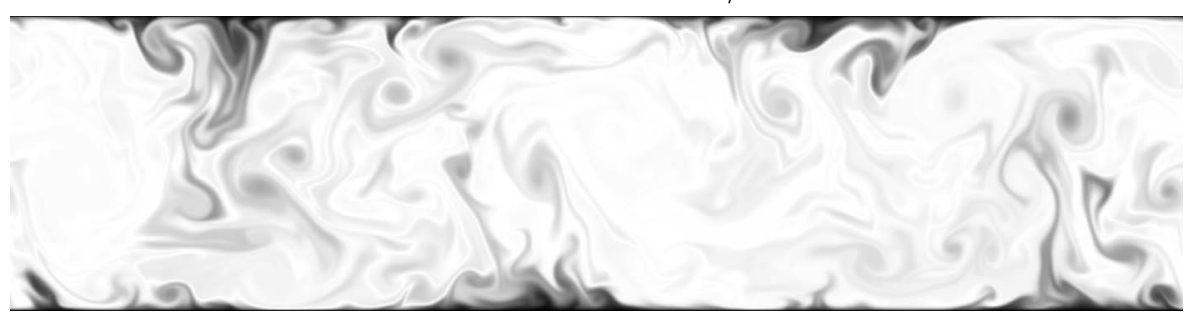

tracer field $\mathrm{Sc}=1$

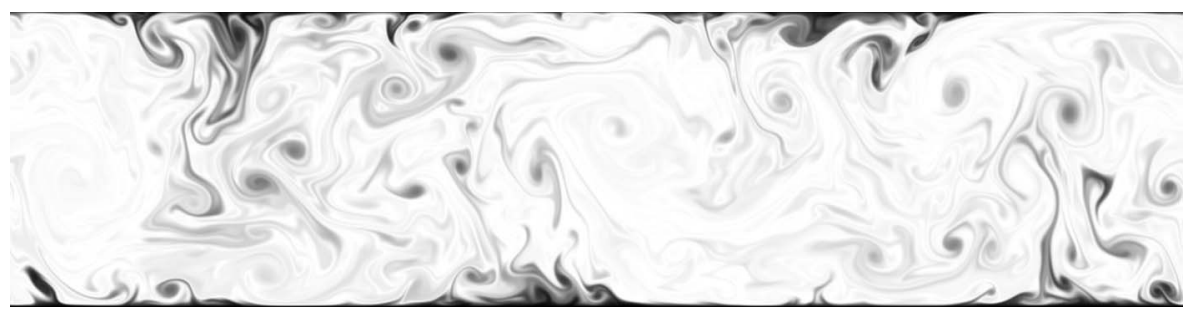

tracer field $\mathrm{Sc}=2$
FIG. 10. The dispersion of a passive tracer for different Schmidt numbers, with the tracer injected at both the upper and lower walls at a constant rate. The absolute value of the concentration of the tracer material is represented by the gray-scale coloring. In the upper panel, a snapshot of the vorticity at the same time is given. is presumed to be rather slow and does not play a significant role in the present simulations. For the second mechanism, the tracer material needs to be contained in a patch with vorticity of the same sign as the dominant large-scale vortex. The large-scale vortex can merge with this patch of vorticity and capture the associated tracer material. In a circulation cell where no strong vortex is present, we observe many small-scale vortices that contain tracer material.

In boundary points where $\omega=0$, the flow separates from the wall, and these points at the boundary have shown to play an important role in tracer removal during the dipole-

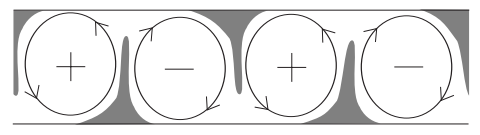

FIG. 11. Schematic representation of the dispersion tracer material (gray) by the domain-sized recirculation cells, where the tracer material is being injected at the upper and lower walls. The sign of the circulation of the cells is denoted by the plus and minus signs, while the arrows give the rotation direction. wall collision, see Ref. 24 for a more elaborate discussion. For our purpose, we will have a closer look on the vorticity and vorticity influx (or vorticity production) at the stationary flat no-slip boundary for 2D turbulence ${ }^{43,44}$ and, in particular, the presence of points with $\omega=0$ at the walls. In Fig. 12, a

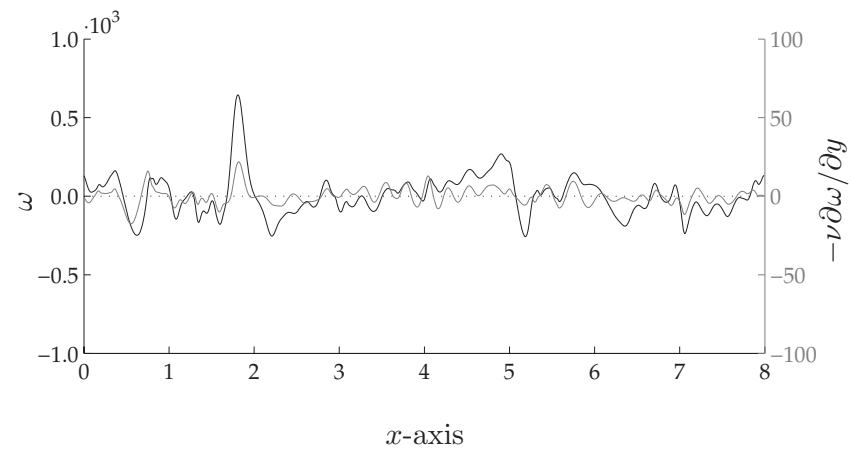

FIG. 12. Values of vorticity $\omega$ (black) and vorticity production $-\nu \partial \omega / \partial y$ (gray) along the wall $(y=-1)$ for $\mathrm{Re}=10000$. The corresponding vorticity field is given in Fig. 10. 


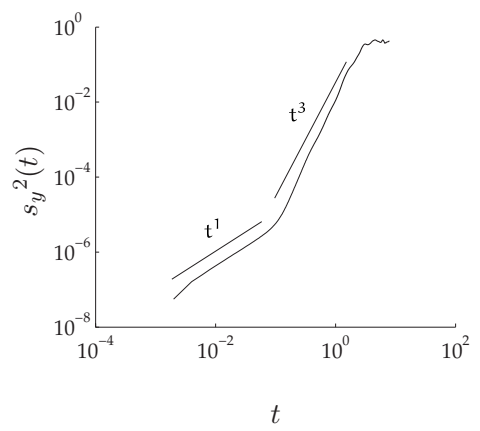

FIG. 13. The tracer variance measured in the wall-normal direction for $\mathrm{Sc}$ $=2$ and $\operatorname{Re} \approx 10000$.

representative distribution of the vorticity $\omega(y=0)$ and vorticity influx $-\nu(\partial \omega / \partial y)_{y=0}$ for a typical run are plotted as a function of $x$, i.e., along the wall $y=-1(\operatorname{Re}=10000)$. There are not so many points where the flow potentially separates (i.e., $\omega=0$ ). There are mainly separation points upstream and downstream of the domain-sized vortices. In the region where these large-scale vortices touch the boundary, no injection of the tracer material occurs. Downstream of the central vortex, the tracer material is injected into the domain in several small bursts and quickly transported into the interior. The upstream tracer material is detached from the wall, but there is no substantial transport away from the wall due to the rotation direction of the domain-sized vortices.

To investigate the rate of tracer dispersion into the domain, the tracer variance in the wall-normal direction is calculated by using

$$
s_{y}^{2}(t)=\iint_{\mathcal{D}}(y-\bar{y})^{2} c(\mathbf{x}, t) d A,
$$

where $\bar{y}$ denotes the $y$ coordinate of the center of mass of the tracer. Only the tracer material that enters through the upper wall is considered in the calculation. In Fig. 13, the results are plotted against time in a double logarithmic graph for the case $\mathrm{Sc}=2$. For short times, $s_{y}^{2}(t) \propto t$, which is related to diffusion of the tracer material. At the wall, the normal velocity is equal to zero, so initially, there is only a diffusive flux of tracer material away from the wall. Thereafter, the rate of dispersion is enormously increased and $s_{v}^{2}(t) \propto t^{3}$. In Fig. 14, the distribution of a passive tracer, which is injected at the upper wall, is plotted for three subsequent times within the range where the dispersion scales as $t^{3}$. The fast dispersion rate might be related to shear flow, but there is no clear correlation. The scaling regime starts with the advection of tracer material around the small-scale vortices near the boundary [Fig. 14(a)]. The boundary-layer vorticity and tracer material are together detached from the wall by the nearby vortex. Vorticity filaments are directly related to thin regions with shear. After the detachment, the vorticity filament rolls up into a vortex and forms together with the impinging primary vortex a dipolar structure. In the center of the dipole, the velocity $v$ is negative (away from the wall) and the shear is virtually zero. Hence, each tracer filament is close to a minimum in the velocity profile $v(x)$, i.e., close to the most negative values of $v(x)$ [see Figs. 14(a) and 14(b)].
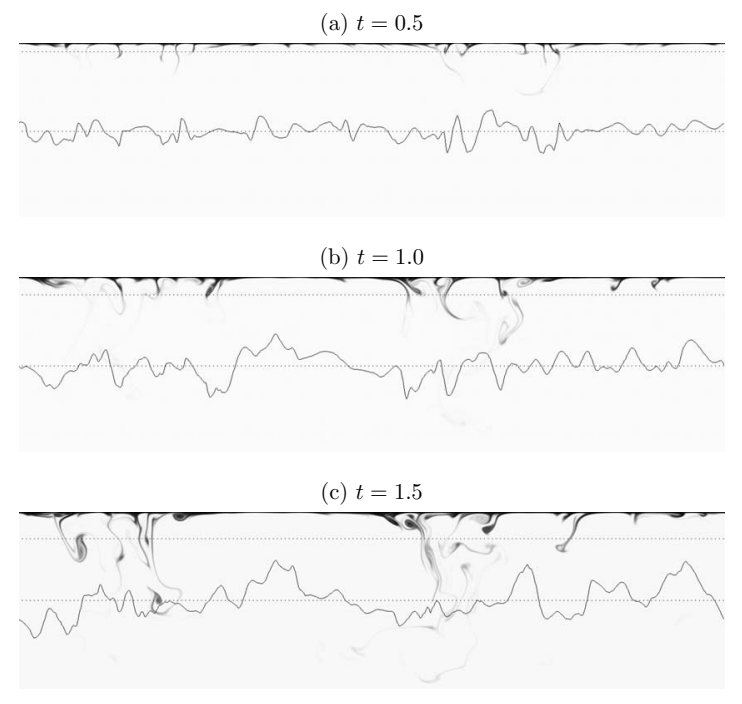

FIG. 14. Snapshots of the tracer field for $\mathrm{Sc}=2$ and $\mathrm{Re} \approx 10000$ in the time regime when dispersion is proportional to $t^{3}$. The tracer is being injected at a constant rate at the upper wall. The velocity profile $v(x)$ is given for the cross sections $y=0.9$ in (a), $y=0.8$ in (b), and $y=0.7$ in (c).

As the dipole containing the tracer material moves away from the wall, it leaves a thin filament of the tracer material. The presence of the tracer material is then no longer correlated to local vertical shear in the flow. The fast increase in the dispersion is limited by the finite size of the domain and the end of the $t^{3}$ regime coincides with the time the first plume of the tracer material reaches the opposite wall.

From the numerical simulations discussed in this section, it can be concluded that despite a uniform release of the passive tracer at the no-slip boundaries, nonuniform transport of the passive tracer away from the boundaries occurs. This is a direct result of the emergence of large-scale vortices in the channel domain.

\section{ENSTROPHY AND TRACER VARIANCE SPECTRA}

Simulation 4A4 is used to investigate the enstrophy and tracer variance spectra. The settings for this simulation are given in Table IV. To obtain spectra of the tracer variance, the tracer material is injected in such a way that a statistically stationary state can be reached. Suppose the fluid is at rest and a mean linear concentration gradient is introduced, with inflow of passive tracer at the upper wall and outflow at the lower wall. In order to mimic this flow, we write the concentration as

$$
c(\mathbf{x}, t)=\mathbf{G} \cdot \mathbf{x}+c^{\prime}(\mathbf{x}, \mathbf{t}),
$$

i.e., with a fixed gradient and a fluctuating part $c^{\prime}(\mathbf{x}, t)$. Here, $\mathbf{G}=\left(0, G_{y}\right)$, i.e., the tracer gradient is perpendicular to the

TABLE IV. Settings and resolution used to solve the tracer dispersion in a

\begin{tabular}{|c|c|c|c|c|c|c|c|}
\hline & & $K$ & $M$ & $\Delta t$ & $F_{\omega}$ & $\tau$ & $\mathbf{k}_{f}$ \\
\hline$\nu$ & $1 \times 10^{-4}$ & 2048 & 512 & $4 \times 10^{-5}$ & 8 & $1 \times 10^{-2}$ & {$[7 \pi, 9 \pi]$} \\
\hline$\kappa$ & $0.5 \times 10^{-4}$ & 2048 & 512 & $4 \times 10^{-5}$ & $\ldots$ & $\ldots$ & $\ldots$ \\
\hline
\end{tabular}
turbulent velocity field. 
variational part of the concentration, $c^{\prime}(\mathbf{x}, t)$

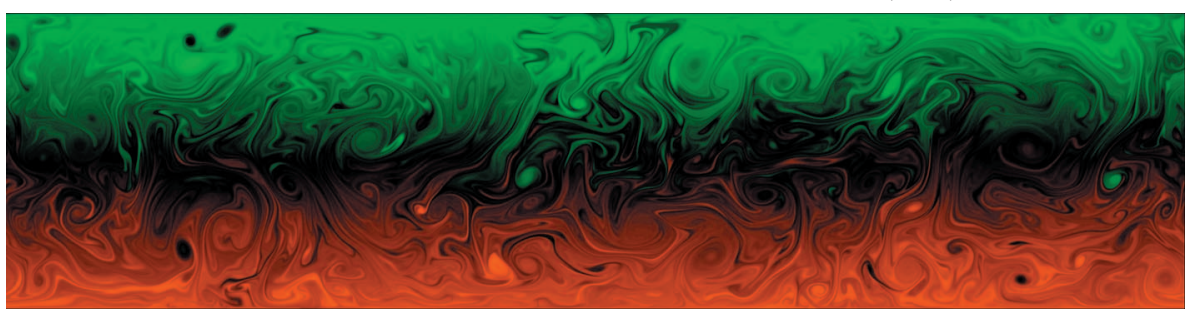

concentration, $c(\mathbf{x}, t)$

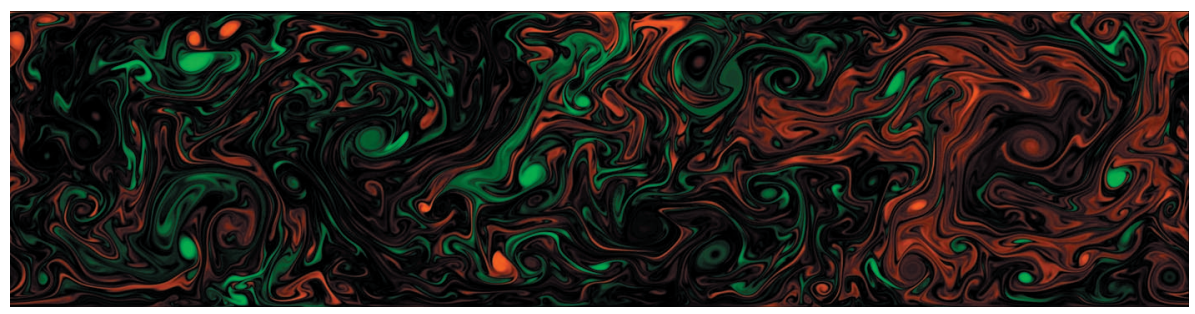

vorticity field

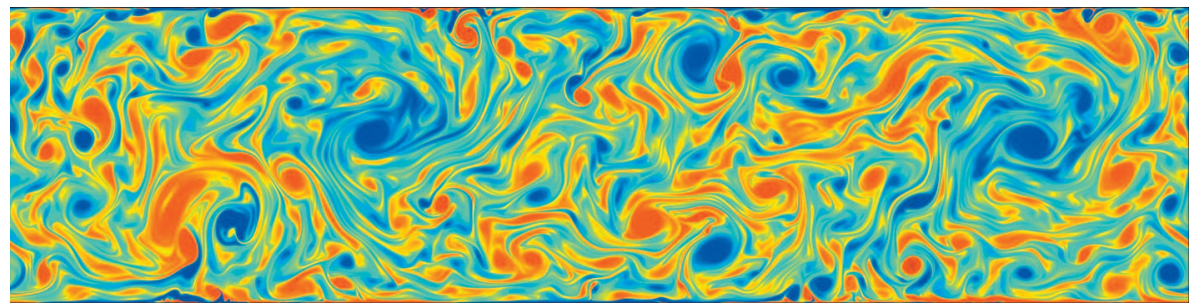

FIG. 15. (Color) The concentration and the fluctuating part of the concentration of the simulations where tracer is injected due to a fixed mean gradient. The red colors depict positive values of the tracer concentration and the red colors depict negative values. The vorticity field at the same time is also plotted, where the red and blue colors represent positive and negative vorticities, respectively. wall. The fluctuations in the concentration are then governed by

$$
\frac{\partial c^{\prime}}{\partial t}+(\mathbf{u} \cdot \nabla) c^{\prime}=\kappa \nabla^{2} c^{\prime}-G_{y} v
$$

where the last term on the right-hand side acts as a source term. At the wall, we apply a no-flux condition for the fluctuating part of the concentration. This kind of tracer forcing was used earlier by Yeung et $a l^{45}$ to investigate the effect of the Schmidt number on turbulent transport and by Brethouwer et $a l .{ }^{46}$ to study the alignment of tracer gradients in $3 \mathrm{D}$ turbulence.

Typical snapshots from this simulation are displayed in Fig. 15. The Reynolds number based on the rms velocity is $\mathrm{Re} \approx 20000$ and $\mathrm{Sc}=2$. In the statistically steady state, $c^{\prime}(\mathbf{x}, t)$ reveals a gradient in the $y$ direction opposite to the fixed mean gradient. The absolute concentration can be obtained by adding the fixed mean tracer gradient, $c^{\prime}(\mathbf{x}, t)$ $+G_{y} y$. Hence, there is no mean gradient in the absolute concentration visible.

Spectra of the enstrophy and tracer variance are calculated along a line analogous to the longitudinal and transverse spectra of the velocity, see Sec. V. For instance, for the enstrophy spectra, we use the Fourier components $\widetilde{\omega}\left(k_{x}, y\right)$ to calculate the one-dimensional enstrophy spectra, $F_{\Omega}\left(k_{x}, y\right)$ $=\frac{1}{2}\left|\omega\left(k_{x}, y\right)\right|^{2}$. The spectra are determined near the wall for $y= \pm 0.99$ (averaged over both lines) and in the interior of the domain at $y=0$, and both spectra are shown in Fig. 16. In the direct enstrophy cascade range, a $k^{-1}$ scaling is expected for the enstrophy and tracer variance spectrum. However, in the interior, the enstrophy spectrum has a slope steeper than -2.4 . The tracer spectrum is less steep in this region with a slope close to -2.1 . Tracer diffusion acts on a larger wave number than vorticity diffusion as $\mathrm{Sc}=2$. Near the wall, the enstrophy spectrum has a slope close to -1.7 , thus less steep than in the interior of the domain. At larger wave numbers, there is no strong exponential decay related to the viscous dissipation of enstrophy. The injection of enstrophy at the no-slip wall seems to have a major contribution at scales close to the boundary-layer thickness. (a) interior $(y=0)$

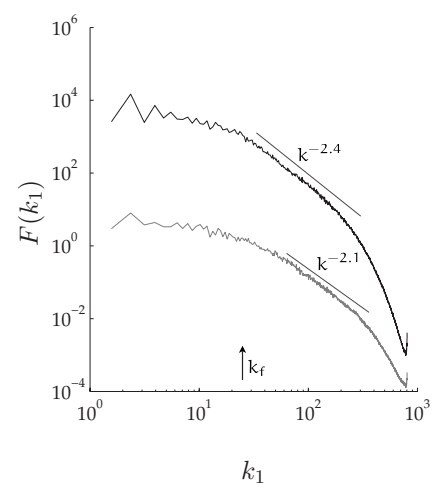

(b) wall region $(y= \pm 0.99)$

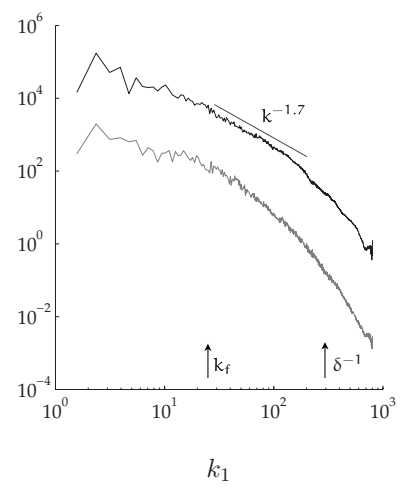

FIG. 16. One-dimensional vorticity (black) and tracer variance spectra (gray), calculated along a line in the interior (a) and near the no-slip wall (b). 

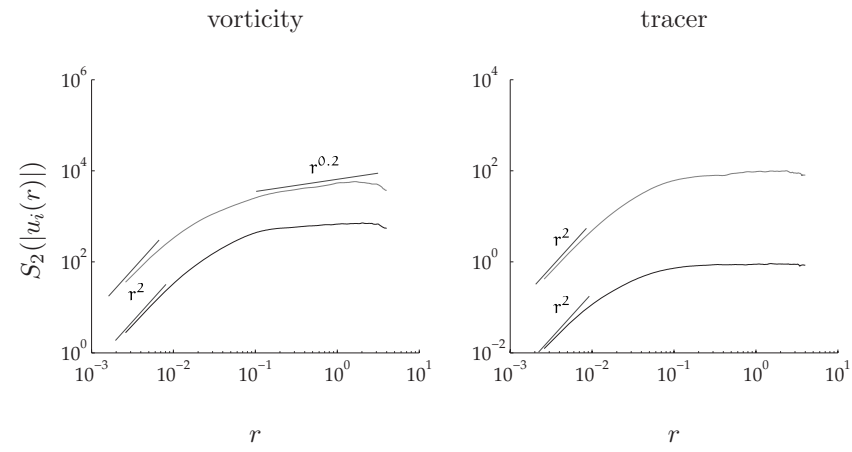

FIG. 17. Second-order structure functions of the vorticity and tracer concentration, calculated along a line in the interior (black) and near the wall (gray).

To investigate this behavior of the spectra more closely, we turn to the second-order vorticity and tracer variance structure functions. The structure function of order $n$ for the vorticity is defined by

$$
S_{n}[\omega(r)]=\left\langle[\omega(x)-\omega(x+r)]^{n}\right\rangle,
$$

where $r$ is the separation and $\langle\cdot\rangle$ denotes taking the average. Averaging is performed both in time (five eddy turnover times) and over the homogeneous periodic direction. The structure function for the tracer concentration is governed by a similar expression. It was argued by Benzi et al. ${ }^{47}$ that if the vorticity structure function scales as $S_{2}[\omega(r)] \sim r^{2 g}$, the enstrophy spectrum should scale as $\Omega(k) \sim k^{-(1+2 g)}$. The power spectrum of the enstrophy is related to the fractal dimension of an isovorticity line, $D_{\omega}$, with $D_{\omega}=2-g$ for 0 $\leqslant g \leqslant 1$. If the isovorticity has a fractal dimension equal to 2 , the isovorticity lines densely fill the whole fluid. In this case, the Kraichnan spectrum is retrieved, $\Omega(k) \sim k^{-1}$. This is the most chaotic situation and the small-scale statistics of the vorticity is equivalent to that of a passive scalar, which has a $k^{-1}$ scaling. If the isovorticity lines are smooth with $D_{\omega}=1$, the enstrophy spectrum scales like $k^{-3}$. Benzi et al. ${ }^{47}$ then argued that if the flow is dominated by coherent vortices, which corresponds to a fractal dimension of $D_{\omega} \approx 1$, the energy spectrum in the enstrophy cascade range should be steeper than -3 .

In Fig. 17, the second-order structure functions for the vorticity and the passive tracer are plotted. For large separations, the tracer variance structure functions are nearly flat, which indicates that the fractal dimension here is close to $D_{c}=2$. For smaller separations, the isoconcentration lines are more smooth and hence the slope of the structure function is expected to be steeper, with $2 g=2$. In the interior, the vorticity structure functions are also nearly flat for large separations, which relates to a fractal dimension of $D_{\omega}=2$. In this range, the vorticity is thus acting like a passive tracer. Near the wall, the structure function does not become entirely flat but has a slope of $2 g=0.2$. The fractal dimension of $D_{\omega}$ $=1.9$ reveals that there is some intermittency in the enstrophy cascade range near the wall. Paret $e t a l .{ }^{48}$ measured a scaling exponent ranging from -0.05 to 0.15 for the vorticity structure functions $S_{n}[\omega(r)]$, with $2 \leqslant n \leqslant 10$ (and $n$ even), in laboratory experiments. However, the presence of the bottom drag makes it uncertain how well these experiments relate to our simulations.

\section{SUMMARY AND DISCUSSION}

We have considered 2D turbulence in a periodic channel driven by a large-scale forcing. The type of flow that develops strongly depends on the aspect ratio of the domain. For small aspect ratios, $\gamma=1$ and 2, a strong unidirectional channel flow is present. Without any applied pressure gradient over the channel length, the channel flow spontaneously develops, much like the spontaneous spin up in a square bounded domain observed in previous studies. ${ }^{11,29}$ For the larger aspect ratios of $\gamma=4$, an array of circulation cells of alternating rotation appears.

There is, however, a large difference between the patterns observed in decaying and in forced turbulence. For forced turbulence, the vorticity field is characterized by vortices of approximately the forcing scale, while the stream function reveals a clear organization into the domain-sized circulation cells. The circulation cells are either caused by a strong vortex that is somewhat smaller than the domain size or by a clustering of like-signed smaller vortices. The strong large vortices can be destabilized by the smaller vortices, which are created by the forcing mechanism or by detachment of wall-generated vorticity.

Tracer material that is uniformly injected at the wall initially diffuses slowly into the interior and is then efficiently injected into the domain due to advective transport by vortices that are located near the wall. This transport away from the boundaries occurs rather nonuniformly with strong injections at certain locations along the boundary only. Thereafter, the tracer is rapidly advected toward the opposite wall due to the large circulation cells. After the diffusive stage, the tracer dispersion is fast, with the squared tracer variance increasing as $t^{3}$. Nearly no tracer material enters the strong domainsized vortices. In the regions of the domain where such a vortex is not present, wall-generated vortices can enter and take the tracer material with them.

The tracer material that is injected at the wall can be considered as a marker for wall-generated vorticity, certainly for the case wherein the Schmidt number is larger than unity. The simulations on the removal of tracer material located near the boundary by the dipole-wall collision revealed that this tracer material becomes trapped inside the wallgenerated vortices. ${ }^{24}$ The fast dispersion of tracer throughout the domain illustrates that the wall-generated vorticity structures also enter the entire domain. This reinforces the importance of the no-slip walls as a source of small-scale vorticity structures as suggested by Clercx and van Heijst. ${ }^{16}$

\section{ACKNOWLEDGMENTS}

One of the authors (W.K.) was supported by the Computational Science program (Project No. 635.000.002) with financial aid from the Netherlands Organisation for Scientific Research (NWO). This program is funded by the Netherlands Organisation for Scientific Research (NWO) and Technology Foundation (STW) under Innovational Research In- 
TABLE V. Decay rate for the gravest Stokes modes on a periodic-channel domain with no-slip walls at $y$ $= \pm 1$.

\begin{tabular}{|c|c|c|c|c|c|c|c|c|}
\hline \multirow[b]{2}{*}{ Symmetry } & \multicolumn{2}{|c|}{$k=0$} & \multicolumn{2}{|c|}{$k=\pi / 4$} & \multicolumn{2}{|c|}{$k=\pi / 2$} & \multicolumn{2}{|c|}{$k=\pi$} \\
\hline & $\mu$ & $\sigma$ & $\mu$ & $\sigma$ & $\mu$ & $\sigma$ & $\mu$ & $\sigma$ \\
\hline$A$ & $\frac{1}{2} \pi$ & -2.47 & $\cdots$ & $\cdots$ & $\cdots$ & $\cdots$ & $\cdots$ & $\cdots$ \\
\hline$S$ & $\pi$ & -9.87 & 2.970 & -9.44 & 2.642 & -9.45 & 2.179 & -14.62 \\
\hline$A$ & $\frac{3}{2} \pi$ & -22.21 & 4.449 & -20.41 & 4.336 & -21.27 & 4.051 & -26.28 \\
\hline
\end{tabular}

centives Scheme Grant No. 06239. This work was sponsored by the Stichting Nationale Computerfaciliteiten (National Computing Facilities Foundation, NCF) for the use of supercomputer facilities.

\section{APPENDIX: SOLUTIONS OF THE STOKES EQUATION ON A PERIODIC CHANNEL DOMAIN}

Solutions of the Stokes equation describe the behavior of the decaying turbulent flows in the long-time limit. To investigate the resemblance between the domain-sized structures observed in forced and decaying 2D turbulence and the Stokes modes, we now discuss the Stokes modes in more detail for a periodic-channel domain. The Stokes equation reads

$$
\frac{\partial \omega}{\partial t}=\nu \nabla^{2} \omega \text { in } \mathcal{D} .
$$

Solutions of this equation vary for different geometries of the domain and can be found by separation of variables, i.e., write the solution as $\omega(\mathbf{x}, t)=T(t) \bar{\omega}(\mathbf{x})$. The time-dependent part is then given by $T(t)=\exp (\nu \sigma t)$, where $\sigma$ is a constant yet to be determined. The spatial part of the solutions, $\bar{\omega}(\mathbf{x})$, is governed by

$$
\nabla^{2} \bar{\omega}-\sigma \bar{\omega}=0 \quad \text { in } \mathcal{D} .
$$

The spatial solution depends on the applied boundary conditions. In case of the 2D periodic channel, the assume that the $x$-dependent part of the solution can be written as a Fourier series. Equation (9) can then be reformulated as

$$
\frac{\partial^{2} \bar{\omega}}{\partial y^{2}}+\mu^{2} \bar{\omega}=0,
$$

where we have introduced $\mu^{2}=-\sigma-k^{2}$, with $k$ the wave number of the Fourier polynomial. Two separate sets of solutions can be determined, the first contains all the Stokes modes that are symmetric in $y$. Without loss of generality, we assume that the no-slip walls are located at $y= \pm 1$. The symmetric solutions are then given by the vorticity field:

$$
\bar{\omega}(\mathbf{x})=-A\left(\mu^{2}+k^{2}\right) \cos (\mu y) \exp (i k x)
$$

and the accompanying velocity field is

$$
\begin{aligned}
& \bar{u}(\mathbf{x})=A \mu[\sin (\mu y)-(\sin \mu / \sinh k) \sinh (k y)] \exp (i k x), \\
& \bar{v}(\mathbf{x})=A i k[\cos (\mu y)-(\cos \mu / \cosh k) \cosh (k y)] \exp (i k x) .
\end{aligned}
$$

The complex constant $A$ determines the phase of the Fourier polynomial and the amplitude of the Stokes modes. The obtained velocity field is only divergence-free when

$$
\mu \tan \mu=-k \tanh k,
$$

which provides the value for $\mu$ when $k$ is specified.

The decay rate of the Stokes modes then follows from $\sigma=-k^{2}-\mu^{2}$. The channel length, equal to $2 L$, prescribes the possible wave numbers $k$ in the $x$ direction. For instance, $k$ $=\pi / L$ is the gravest nonzero wave number. The values $\mu$ and $\sigma$ for the gravest Stokes modes are given in Table V for a channel with $L=4$. Any combination of $k$ and $\mu$ results in a negative $\sigma$, which is required for the time-dependent part to remain finite (and decaying) in time. The gravest mode with the smallest $k$ and $\mu$ decays at the slowest rate.

The solutions antisymmetric in $y$ are given by

$$
\bar{\omega}(\mathbf{x})=-A\left(\mu^{2}+k^{2}\right) \sin (\mu y) \exp (i k x),
$$

and the velocity field by

$$
\begin{aligned}
& \bar{u}(\mathbf{x})=-A \mu[\cos (\mu y)-(\cos \mu / \cosh k) \cosh (k y)] \exp (i k x), \\
& \bar{v}(\mathbf{x})=A i k[\sin (\mu y)-(\sin \mu / \sinh k) \sinh (k y)] \exp (i k x) .
\end{aligned}
$$

The values for $\mu$ now have to satisfy the relation

$$
\mu \cot \mu=k \operatorname{coth} k,
$$

which, again, is required for the velocity field to be divergence free. The complete solution to the Stokes equation (8) can be composed by using both the symmetric and the antisymmetric Stokes modes.

For the special case $k=0$, the symmetric solution reduces to

$$
\begin{aligned}
& \bar{\omega}(\mathbf{x})=-A \mu^{2} \cos (\mu y), \\
& \bar{u}(\mathbf{x})=A \mu \sin (\mu y), \\
& \bar{v}(\mathbf{x})=0 .
\end{aligned}
$$

The no-slip constraint at $y= \pm 1$ simply yields that $\mu=n \pi$. The antisymmetric solution reads

$$
\bar{\omega}(\mathbf{x})=-A \mu^{2} \sin (\mu y),
$$




$$
\begin{aligned}
& \bar{u}(\mathbf{x})=-A \mu \cos (\mu y), \\
& \bar{v}(\mathbf{x})=0 .
\end{aligned}
$$

where $\mu= \pm\left(n+\frac{1}{2}\right) \pi$ (negative values for $\mu$ are only relevant for the antisymmetric solutions). The decay rate follows from $\sigma=-\mu^{2}$, which is always negative.

${ }^{1}$ R. H. Kraichnan, "Inertial ranges in two-dimensional turbulence," Phys. Fluids 10, 1417 (1967).

${ }^{2}$ G. K. Batchelor, "Computation of the energy spectrum in two-dimensional turbulence," Phys. Fluids 12, II-233 (1969).

${ }^{3}$ C. E. Leith, "Minimum enstrophy vortices," Phys. Fluids 27, 1388 (1984).

${ }^{4}$ Y. Couder, J. M. Chomaz, and M. Rabaud, "On the hydrodynamics of soap films," Physica D 37, 384 (1989).

${ }^{5}$ H. Kellay and W. I. Goldburg, "Two-dimensional turbulence: A review of some recent experiments," Rep. Prog. Phys. 65, 845 (2002).

${ }^{6}$ J. B. Flór and G. J. F. van Heijst, "Stable and unstable monopolar vortices in a stratified fluid," J. Fluid Mech. 311, 257 (1996).

${ }^{7}$ S. D. Danilov and D. Gurarie, "Quasi-two-dimensional turbulence," Phys. Usp. 43, 863 (2000).

${ }^{8} \mathrm{~S}$. Li and D. Montgomery, "Decaying two-dimensional turbulence with rigid walls," Phys. Lett. A 218, 281 (1996).

${ }^{9} \mathrm{~S}$. Li and D. Montgomery, "Inverse cascades of angular momentum," J. Plasma Phys. 56, 615 (1996).

${ }^{10} \mathrm{~S}$. Li, D. Montgomery, and W. B. Jones, "Two-dimensional turbulence with rigid circular walls," Theor. Comput. Fluid Dyn. 9, 167 (1997).

${ }^{11}$ H. J. H. Clercx, S. R. Maassen, and G. J. F. van Heijst, "Spontaneous spin-up during the decay of $2 \mathrm{D}$ turbulence in a square container with rigid boundaries," Phys. Rev. Lett. 80, 5129 (1998).

${ }^{12}$ H. J. H. Clercx, S. R. Maassen, and G. J. F. van Heijst, "Decaying twodimensional turbulence in square containers with no-slip or stress-free boundaries," Phys. Fluids 11, 611 (1999).

${ }^{13}$ S. R. Maassen, H. J. H. Clercx, and G. J. F. van Heijst, "Decaying quasi-2D turbulence in a stratified fluid with circular boundaries," Europhys. Lett. 46, 339 (1999).

${ }^{14}$ S. R. Maassen, H. J. H. Clercx, and G. J. F. van Heijst, "Self-organization of quasi-two-dimensional turbulence in stratified fluids in square and circular containers," Phys. Fluids 14, 2150 (2002).

${ }^{15}$ S. R. Maassen, H. J. H. Clercx, and G. J. F. van Heijst, "Self-organization of decaying quasi-2D turbulence in stratified fluids in rectangular containers," J. Fluid Mech. 495, 19 (2003).

${ }^{16}$ H. J. H. Clercx and G. J. F. van Heijst, "Energy spectra for decaying 2D turbulence in a bounded domain," Phys. Rev. Lett. 85, 306 (2000).

${ }^{17}$ D. M. Fratantoni, A. S. Bower, W. E. Johns, and H. Peters, "Somali current rings in the eastern Gulf of Aden," J. Geophys. Res. 111, C09039, DOI: 10.1029/2005JC003338 (2006).

${ }^{18}$ G. Buffoni, P. Falco, A. Griffa, and E. Zambianchi, "Dispersion processes and residence times in a semi-enclosed basin with recirculating gyres: An application to the Tyrrhenian sea," J. Geophys. Res. 102, 699 (1997).

${ }^{19}$ P. Falco, A. Griffa, P.-M. Poulain, and E. Zambianchi, "Transport properties in the Adriatic Sea as deduced from drifter data," J. Phys. Oceanogr. 30, 2055 (2000)

${ }^{20} \mathrm{~K}$. Schneider and M. Farge, "Decaying two-dimensional turbulence in a circular container," Phys. Rev. Lett. 95, 244502 (2005).

${ }^{21}$ G. H. Keetels, U. D’Ortana, W. Kramer, H. J. H. Clercx, K. Schneider, and G. J. F. van Heijst, "Fourier spectral and wavelet solvers for the incompressible Navier-Stokes equations with volume-penalization: Convergence of a dipole wall collision,” J. Comput. Phys. 227, 919 (2007).

${ }^{22} \mathrm{O}$. Daube, "Resolution of 2D Navier-Stokes equations in velocityvorticity form by means of an influence matrix technique," J. Comput. Phys. 103, 402 (1992).
${ }^{23}$ H. J. H. Clercx, "A spectral solver for the Navier-Stokes equations in the velocity-vorticity formulation for flows with two non-periodic directions," J. Comput. Phys. 137, 186 (1997).

${ }^{24}$ W. Kramer, "Dispersion of tracers in two-dimensional bounded turbulence," Ph.D. thesis, Eindhoven University of Technology, 2007.

${ }^{25}$ D. K. Lilly, "Numerical simulation of two-dimensional turbulence," Phys. Fluids 12, II-240 (1969).

${ }^{26}$ M. E. Maltrud and G. K. Vallis, "Energy spectra and coherent structures in forced 2D and beta-plane turbulence," J. Fluid Mech. 228, 321 (1991).

${ }^{27}$ K. G. Oetzel and G. K. Vallis, "Strain, vortices, and the enstrophy inertial range in two-dimensional turbulence," Phys. Fluids 9, 2991 (1997).

${ }^{28}$ H. J. H. Clercx, A. H. Nielsen, D. J. Torres, and E. A. Coutsias, "Twodimensional turbulence in square and circular domains with no-slip walls," Eur. J. Mech. B/Fluids 20, 557 (2001).

${ }^{29}$ D. Molenaar, H. J. H. Clercx, and G. J. F. van Heijst, "Angular momentum of forced 2D turbulence in a square no-slip domain," Physica D 196, 329 (2004).

${ }^{30}$ G. J. F. van Heijst, P. A. Davies, and R. G. Davis, "Spin-up in a rectangular container," Phys. Fluids A 2, 150 (1990).

${ }^{31}$ J. A. van de Konijnenberg, H. I. Andersson, J. T. Billdal, and G. J. F. van Heijst, "Spin-up in a rectangular tank with low angular velocity," Phys. Fluids 6, 1168 (1994).

${ }^{32}$ M. G. Wells, H. J. H. Clercx, and G. J. F. van Heijst, "Vortices in oscillating spin-up," J. Fluid Mech. 573, 339 (2007).

${ }^{33}$ S. A. Orszag, M. Israeli, and M. O. Deville, "Boundary conditions for incompressible flows," J. Sci. Comput. 1, 75 (1986).

${ }^{34}$ J. A. van de Konijnenberg, J. B. Flór, and G. J. F. van Heijst, "Decaying quasi-two-dimensional viscous flow on a square domain," Phys. Fluids 10, 595 (1998).

${ }^{35}$ P. F. Batcho and G. E. Karniadakis, "Generalized Stokes eigenfunctions: A new trial basis for the solution of incompressible Navier-Stokes equations," J. Comput. Phys. 115, 121 (1994).

${ }^{36}$ G. H. Keetels, "Fourier spectral computation of geometrically confined two-dimensional flows," Ph.D. thesis, Eindhoven University of Technology, 2008.

${ }^{37}$ L. M. Smith and V. Yakhot, "Finite-size effects in forced two-dimensional turbulence," J. Fluid Mech. 274, 115 (1994).

${ }^{38}$ U. Frisch and P. L. Sulem, "Numerical simulation of the inverse energy cascade in two-dimensional turbulence," Phys. Fluids 27, 1921 (1984).

${ }^{39}$ B. Legras, P. Santangelo, and R. Benzi, "High-resolution numerical experiments for forced two-dimensional turbulence," Europhys. Lett. 5, 37 (1988).

${ }^{40}$ A. Babiano, B. Dubrulle, and P. Frick, "Scaling properties of numerical two-dimensional turbulence," Phys. Rev. E 52, 3719 (1995).

${ }^{41}$ J. Sommeria, "Experimental study of the two-dimensional inverse energy cascade in a square box," J. Fluid Mech. 170, 139 (1986).

${ }^{42}$ J. Paret and P. Tabeling, "Experimental observation of the twodimensional inverse energy cascade,” Phys. Rev. Lett. 79, 4162 (1997).

${ }^{43}$ M. J. Lighthill, Introduction to Boundary Layer Theory, edited by L. Rosenhead (Oxford University Press, New York, 1963).

${ }^{44}$ B. R. Morton, "The generation and decay of vorticity," Geophys. Astrophys. Fluid Dyn. 28, 277 (1984).

${ }^{45}$ P. K. Yeung, S. Xu, and K. R. Sreenivasan, "Schmidt number effects on turbulent transport with uniform mean scalar gradient," Phys. Fluids 14, 4178 (2002).

${ }^{46}$ G. Brethouwer, J. C. R. Hunt, and F. T. M. Nieuwstadt, "Micro-structure and Lagrangian statistics of the scalar field with a mean gradient in isotropic turbulence," J. Fluid Mech. 474, 193 (2003).

${ }^{47}$ R. Benzi, G. Paladin, and A. Vulpiani, "Power spectra in two-dimensional turbulence," Phys. Rev. A 42, 3654 (1990).

${ }^{48}$ J. Paret, M. Jullien, and P. Tabeling, "Vorticity statistics in the twodimensional enstrophy cascade," Phys. Rev. Lett. 83, 3418 (1999). 